ARTICLE

Received 15 May 2014 | Accepted 17 Sep 2014 | Published 22 Oct 2014

DOI: $10.1038 /$ ncomms6288

OPEN

\title{
Dynamic DNA methylation orchestrates cardiomyocyte development, maturation and disease
}

Ralf Gilsbach ${ }^{1, \star}$, Sebastian Preiss ${ }^{1,2, \star}$, Björn A. Grüning 3,4 , Tilman Schnick ${ }^{1,5}$, Lukas Burger ${ }^{6,7}$, Vladimir Benes ${ }^{8}$, Andreas Würch ${ }^{9}$, Ulrike Bönisch ${ }^{9}$, Stefan Günther ${ }^{4}$, Rolf Backofen ${ }^{3}$, Bernd K. Fleischmann ${ }^{10}$, Dirk Schübeler ${ }^{6,11}$ \& Lutz Hein ${ }^{1,12}$

The heart is a highly specialized organ with essential function for the organism throughout life. The significance of DNA methylation in shaping the phenotype of the heart remains only partially known. Here we generate and analyse DNA methylomes from highly purified cardiomyocytes of neonatal, adult healthy and adult failing hearts. We identify large genomic regions that are differentially methylated during cardiomyocyte development and maturation. Demethylation of cardiomyocyte gene bodies correlates strongly with increased gene expression. Silencing of demethylated genes is characterized by the polycomb mark H3K27me3 or by DNA methylation. De novo methylation by DNA methyltransferases 3A/B causes repression of fetal cardiac genes, including essential components of the cardiac sarcomere. Failing cardiomyocytes partially resemble neonatal methylation patterns. This study establishes DNA methylation as a highly dynamic process during postnatal growth of cardiomyocytes and their adaptation to pathological stress in a process tightly linked to gene regulation and activity.

\footnotetext{
${ }^{1}$ Institute of Experimental and Clinical Pharmacology and Toxicology, University of Freiburg, Albertstrasse 25, 79104 Freiburg, Germany. ${ }^{2}$ Hermann Staudinger Graduate School, University of Freiburg, Albertstrasse 21, 79104 Freiburg, Germany. ${ }^{3}$ Bioinformatics Group, Department of Computer Science, University of Freiburg, Georges-Köhler-Allee 106, 79110 Freiburg, Germany. ${ }^{4}$ Pharmaceutical Bioinformatics, Institute of Pharmaceutical Sciences, University of Freiburg, Hermann-Herder-Strasse 9, 79104 Freiburg, Germany. ${ }^{5}$ University Heart Center Freiburg/Bad Krozingen, Department of Congenital Heart Defects and Paediatric Cardiology, University of Freiburg, Hugstetter Strasse 55, 79106 Freiburg, Germany. 6 Friedrich Miescher Institute for Biomedical Research, Maulbeerstrasse 66, 4058 Basel, Switzerland. ${ }^{7}$ Swiss Institute of Bioinformatics, Maulbeerstrasse 66, 4058 Basel, Switzerland. ${ }^{8}$ European Molecular Biology Laboratory, Genomics Core Facility, Meyerhofstraße 1, 69117 Heidelberg, Germany. ${ }^{9}$ Max Planck Institute of Immunobiology and Epigenetics, Stübeweg 51, 79108 Freiburg, Germany. ${ }^{10}$ Institute of Physiology I, Life and Brain Center, University of Bonn, Sigmund-Freud-Straße 25,53127 Bonn, Germany. ${ }^{11}$ University of Basel, Petersplatz 1, 4003 Basel, Switzerland. ${ }^{12}$ BIOSS Centre for Biological Signalling Studies, University of Freiburg, Schänzlestrasse 18, 79104 Freiburg, Germany. ${ }^{\star}$ These authors contributed equally to this work. Correspondence and requests for materials should be addressed to L.H. (email: lutz.hein@pharmakol.uni-freiburg.de).
} 
$\mathrm{D}$ uring its development and postnatal life, the heart has to adapt to diverse physiological and pathophysiological needs. Cardiomyocytes arise from progenitors in early development and mature with limited cell division after birth ${ }^{1,2}$. The transition from pre- to postnatal life demands a number of contractile and metabolic adaptations to facilitate organ growth in the presence of optimal contractile function ${ }^{3-5}$. Due to its limited regenerative capacity, cardiomyocytes respond to these challenges during development and disease with characteristic gene expression programmes. Several epigenetic processes, including microRNAs ${ }^{6}$, chromatin and histone proteins ${ }^{7-9}$ as well as DNA methylation ${ }^{10}$, have been implicated as modulators of cardiac gene expression in development and disease.

DNA methylation is a stable hallmark of cell type identity and is essential for mammalian development ${ }^{11,12}$. It occurs mainly in palindromic $\mathrm{CpG}$ dinucleotides. Whereas most regions of the genome are depleted for $\mathrm{CpGs}$, they are clustered in $\mathrm{CpG}$ islands. CpG islands mark $70 \%$ of annotated mammalian promoters ${ }^{13}$. CpG methylation is essential for proper gene expression, development and genome stability ${ }^{14}$. DNA methylation patterns are maintained during cell division by DNA methyltransferase 1 (DNMT1). De novo DNA methylation is mediated by DNMT3A and DNMT3B ${ }^{15}$. Removal of DNA methylation involves oxidation of 5-methyl-cytosine. The key enzymes for this initial step are the recently discovered ten-eleven translocation enzymes TET1-3 (ref. 16). So far there is limited knowledge about the in vivo time course of DNA methylation pattern establishment in cardiomyocytes ${ }^{17,18}$.

Here we show that DNA methylation is highly dynamic during cardiomyocyte development, postnatal maturation and disease. Demethylated regions in neonatal and adult cardiomyocytes are localized in cell type-specific enhancer regions and in gene bodies of cardiomyocyte genes. Postnatal DNA demethylation correlates with active histone marks and increased gene expression. Repression of demethylated genes is achieved by polycombmediated histone $\mathrm{H} 3 \mathrm{~K} 27$ trimethylation or by de novo methylation by DNA methyltransferases DNMT3A/B. Dynamic DNA methylation is important for the perinatal switch in sarcomere protein isoforms and postnatal cardiomyocyte maturation and adaptation.

\section{Results}

Epigenetic characterization of cardiomyocytes. To adapt to the requirements of the growing organism, cardiomyocytes increase dramatically in size during physiological postnatal growth (Fig. 1a). In response to cardiac injury, for example, during chronic ventricular pressure overload, cardiomyocytes may initiate further pathological growth (Fig. 1a). To provide insight into the dynamics of DNA methylation in development and disease of cardiomyocytes as a prototypical terminally differentiated cell type, we generated DNA methylomes of newborn, adult healthy and adult failing cardiomyocytes.

Since the heart is a complex tissue with cardiomyocytes contributing only $20-30 \%$ of the total cell population ${ }^{19}$, we purified cardiomyocyte nuclei from murine cardiac tissue. An antibody against pericentriolar material 1 (PCM1) $)^{20}$ was used to isolate cardiomyocyte nuclei using flow cytometry (fluorescenceactivated cell sorting (FACS)) or magnetic-assisted nuclei sorting with very high purity ( $>97 \%$; Fig. 1b; Supplementary Fig. 1a-c). To validate the specificity of PCM1 staining, we used transgenic mice with cardiomyocyte-specific histone H2B-mCherry expression (Supplementary Fig. 1d). Isolated cardiac nuclei of these animals and wild-type mice were stained with PCM1 antibody or isotype control IgG (immunoglobulin G) and were analysed by FACS (Supplementary Fig. 1e-g). The overlap of
mCherry and PCM1-positive nuclei was $>95 \%$ confirming that PCM1 specifically stains cardiomyocyte nuclei (Supplementary Fig. 1g). Furthermore, a molecular beacon targeting cardiac troponin T type 2 (Tnnt2) mRNA stained the major fraction of PCM1-positive nuclei (Supplementary Fig. 1h). Analysis of different developmental and disease stages revealed that the proportion of cardiomyocyte nuclei decreased from $68.7 \pm 0.6 \%$ in newborn hearts to $29.7 \pm 0.5 \%$ in adult healthy hearts and to $19.7 \pm 0.7 \%$ in adult failing hearts (mean \pm s.e.m., $n=6$; Fig. 1c; Supplementary Fig. 1i-k). DNA from these purified cardiomyocyte nuclei was bisulfite converted and DNA methylomes were obtained at base-pair resolution using deep sequencing. Three independent experiments with high reproducibility (Supplementary Fig. 2) resulted in high-coverage methylomes (Supplementary Fig. 3; Supplementary Table 1). DNA methylomes from murine embryonic stem (ES) cells ${ }^{21}$ and from total cardiac tissue ${ }^{10}$ were used for comparison.

To gain further insight into the epigenetic landscape, we generated maps for four histone modifications (H3K4me1, H3K4me3, H3K27ac and H3K27me3) from purified cardiomyocyte nuclei (Supplementary Table 2). To generate matching cardiomyocyte-specific transcriptomes, hearts were digested enzymatically, vital cardiomyocytes were sorted by FACS and cardiomyocyte mRNA was subjected to RNA sequencing.

Differential CpG methylation. Visual inspection of two prototypical cardiomyocyte genes, $\alpha$ - and $\beta$-myosin heavy chains (Myh6 and $M y h 7)$, revealed a progressive CpG demethylation in cardiomyocytes purified from newborn as compared with adult and failing hearts (Fig. 1d). Almost complete demethylation of gene body CpGs of Myh6 and Myh7 was apparent in purified cardiomyocyte nuclei but could not be detected in cardiac tissue $^{10}$, which contains several additional cell types (Fig. 1d). To further validate this finding, methylation of $\mathrm{CpGs}$ of the cardiomyocyte-specific gene encoding for the sarcoplasmic reticulum $\mathrm{Ca}^{2+}$ ATPase (SERCA2A, gene Atp2a2) was analysed by pyrosequencing (Supplementary Fig. 4). Atp2a2 methylation levels greatly differed between myocyte and nonmyocyte cell fractions with intermediate levels in cardiac tissue (Supplementary Fig. 4a-d).

In total, we identified 79,655 differentially methylated regions (DMRs) with an average size of $840 \mathrm{bp}$ when comparing adult healthy cardiomyocytes and undifferentiated ES cells (Supplementary Fig. 5). This amounted to $5 \%$ of all CpGs in the mouse genome (Fig. 1e). Ninety percent of these CpGs were hypomethylated and $10 \%$ were hypermethylated in cardiomyocytes versus ES cells (Fig. 1e). Remarkably, a substantial number of CpGs were differentially methylated between newborn and adult cardiomyocytes (6,436 DMRs; Fig. 1e). These data indicate that differential CpG methylation was not restricted to cell type specification during embryonic development, but extended well into the postnatal period of cardiomyocytes. Genomic annotation of cardiomyocyte DMRs showed a predominance for intragenic and $\mathrm{CpG}$ island-flanking regions (Supplementary Fig. 6).

Hypomethylated regions with cardiomyocyte enhancer signature. Previous studies have identified transcription factorbinding sites and histone marks, which are characteristic for enhancers in demethylated regions $s^{10,21,22}$. This prompted us to analyse the enrichment of transcription factor (TF)-binding motifs in DMRs. Demethylated regions in ES cells (Fig. 1f) were significantly enriched $\left(P<10^{-20}\right.$, hypergeometric test $)$ for binding sites of stem cell transcription factors including OCT4 (gene symbol Pouffi), NANOG and others (Supplementary 
a
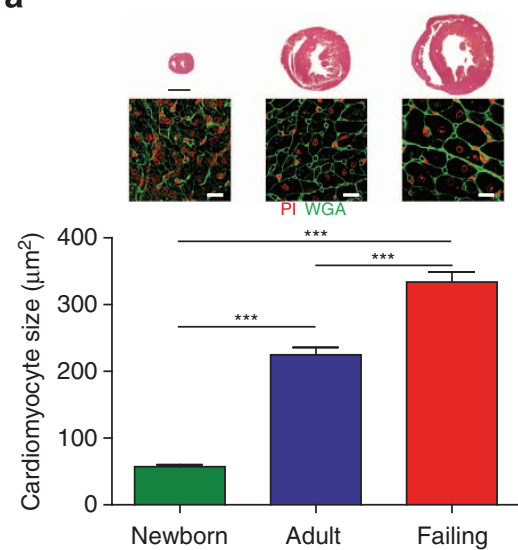

b
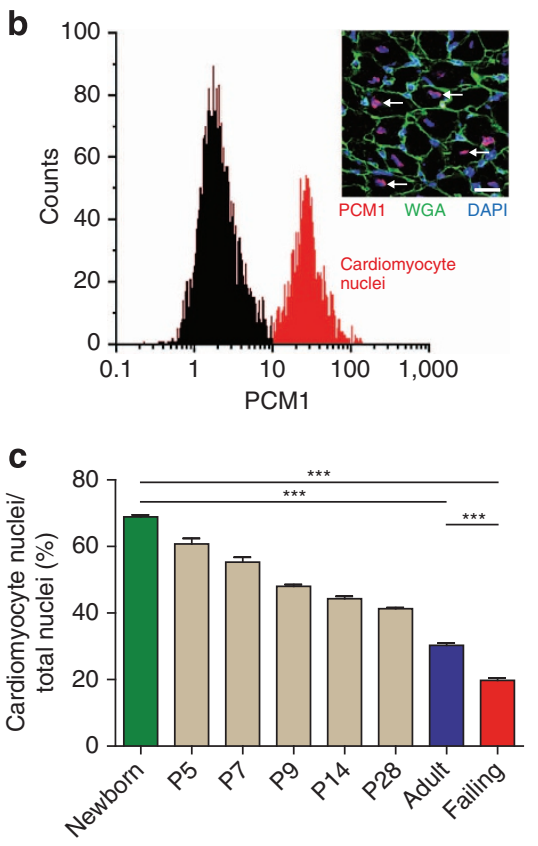

d

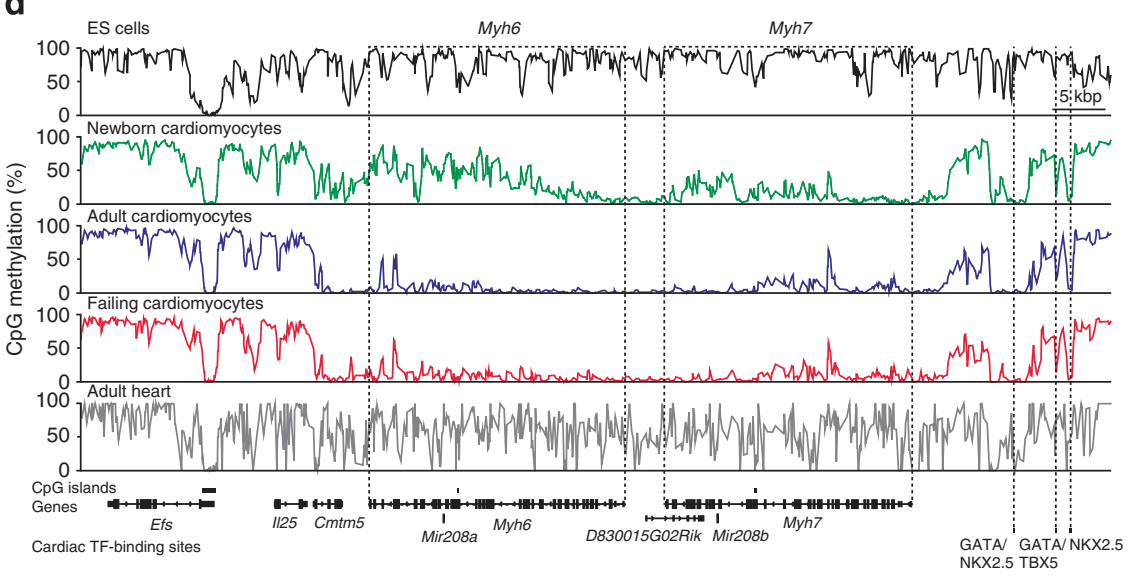

e

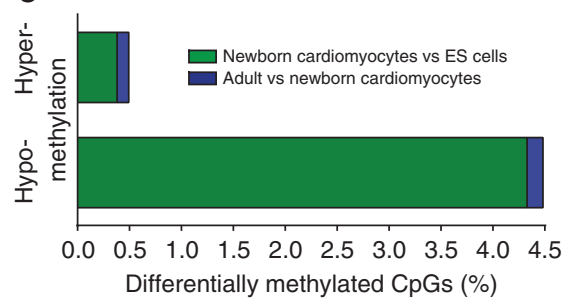

f

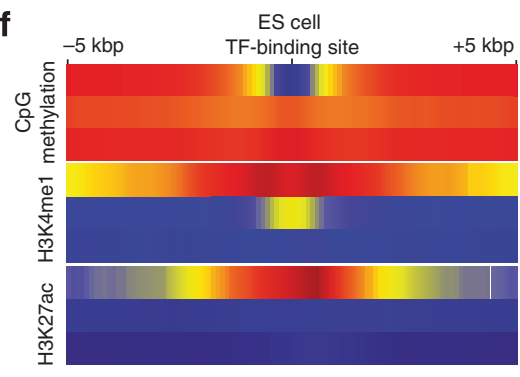

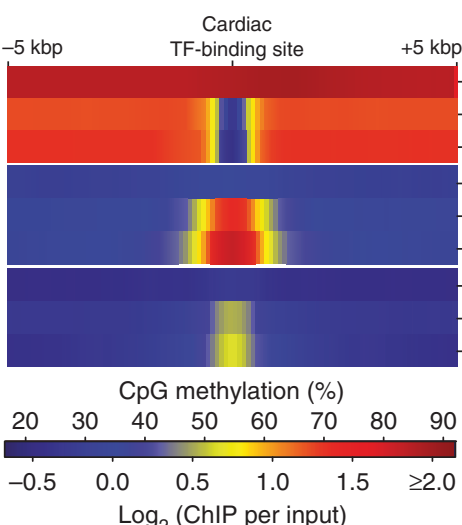

Figure 1 | Analysis of DNA methylation in cardiomyocytes isolated from newborn and adult murine hearts. (a) Hematoxylin-eosin staining (upper panels; scale bar, $1 \mathrm{~mm}$ ) of mouse hearts 1 day after birth (newborn), at 8 weeks of age (adult) and 3 weeks after chronic pressure overload in adult mice (failing). WGA-stained ventricular sections (middle panels; scale bar, $20 \mu \mathrm{m}$ ) to determine cardiomyocyte cross-sectional areas ( $n=10$ hearts per group). Nuclei were stained with propidium iodide (PI). (b) Identification of cardiomyocyte nuclei by PCM1 immunostaining in adult mouse cardiac tissue (insert, arrows; scale bar, $20 \mu \mathrm{m}$ ) and purification of cardiomyocyte nuclei (red) by flow cytometry (histogram). (c) Percentage of cardiomyocyte nuclei in ventricular biopsies identified by PCM1 flow cytometry in newborn, adult healthy and failing hearts and at postnatal days 5-28 ( $n=3-6$ hearts per group). (d) IGV (integrative genomics viewer) traces of CpG methylation of the myosin-6 (Myh6) and myosin-7 (Myh7) gene region in ES cells, newborn, adult and failing cardiomyocytes and in murine heart tissue. (e) Differentially methylated $\mathrm{CpGs}$ during fetal and postnatal development in percent of assessed $\mathrm{CpGs}$. (f) Density of $\mathrm{CpG}$ methylation, histone H3K4me1 and H3K27ac at transcription factor (TF)-binding sites ( $\pm 5 \mathrm{kbp}$ ). Data are shown as mean \pm s.e.m., ${ }^{\star \star \star} P<0.001$, analysis of variance, Bonferroni post hoc test.

Data 1). In contrast, demethylated regions of adult cardiomyocytes were significantly enriched $\left(P<10^{-50}\right.$, hypergeometric test) for TF motifs of known cardiac transcription factors including MEF2C, GATA1-4 and others (Fig. 1f; Supplementary Fig. 7a-c; Supplementary Data 1). These TF included the recently identified master regulators of cellular identity of ES cells or hearts, respectively ${ }^{23}$. In the demethylated state, TF-binding regions were found to be enriched for histone modifications (H3K4me1, H3K27ac), which are typical for active cis-regulatory sites ${ }^{21,24}$ (Fig. 1f). In line with being regulatory, these DMRs were located within distal regulatory regions of genes characteristic for ES cells or cardiomyocytes, respectively (Supplementary Fig. 7d,e).
Genic CpG demethylation affects specific gene programmes. Since many sites of dynamic DNA methylation occurred within genic regions (Supplementary Fig. 6), we explored this aspect in more detail (Fig. 2a,b). At 765 genes, genic DMRs extended to more than $25 \%$ of the entire gene body (Fig. 2a,b). In 78 cases, gene bodies were demethylated in ES cells, yet they were methylated to a higher degree in cardiomyocytes (Fig. 2b, group I; Supplementary Data 2). Gene ontology analysis of this group revealed a predominance of genes involved in embryonic pattern specification and morphogenesis (Supplementary Fig. 8) containing known pluripotency factors like Pou5f1 and Nanog (Fig. 2c; Supplementary Data 2). However, the inverse case with 687 genes, which were demethylated in cardiomyocytes versus ES 
a

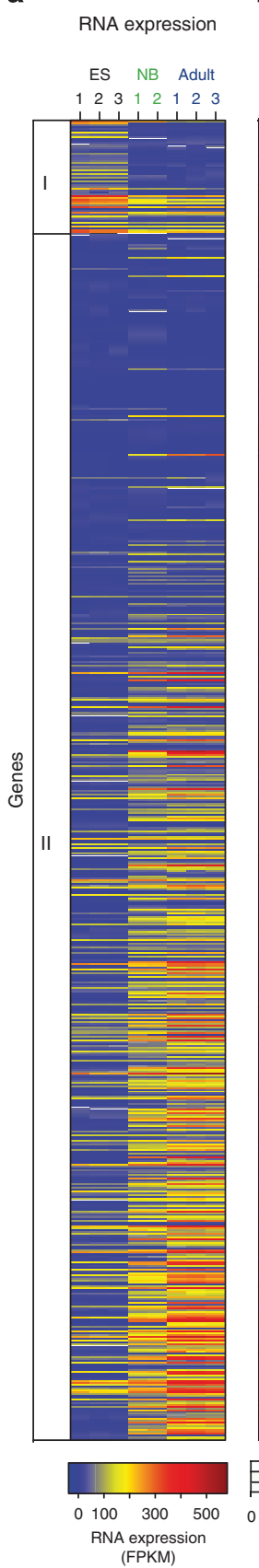

b
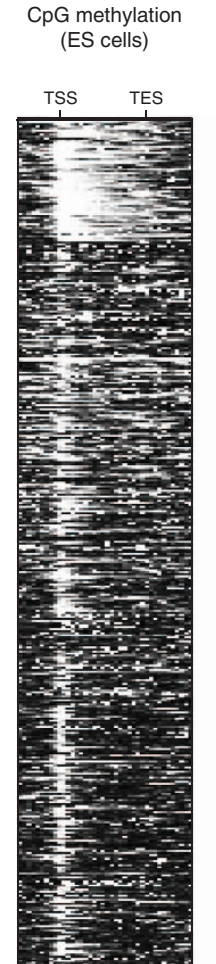
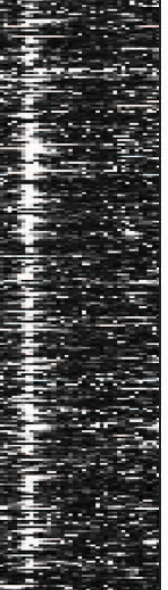

CpG methylation (newborn cardiomyocytes) TSS TES

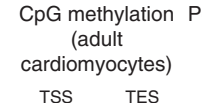

Postnatal CpG methylation

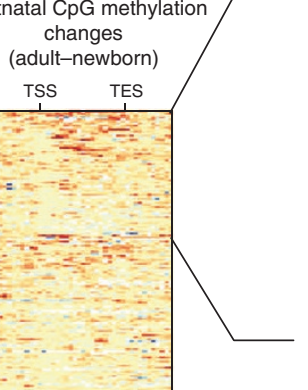

C

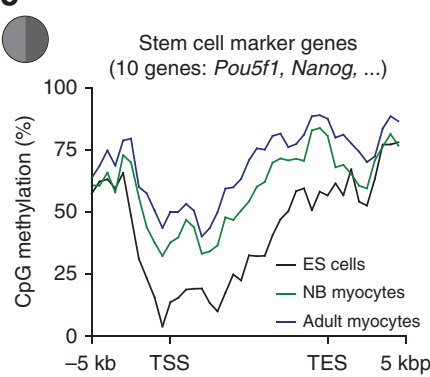

d
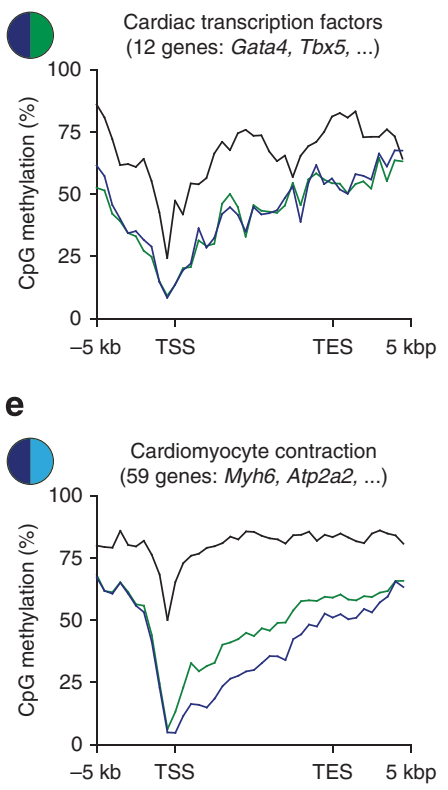

f

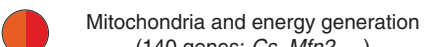
(140 genes: Cs, Mfn2, ...)
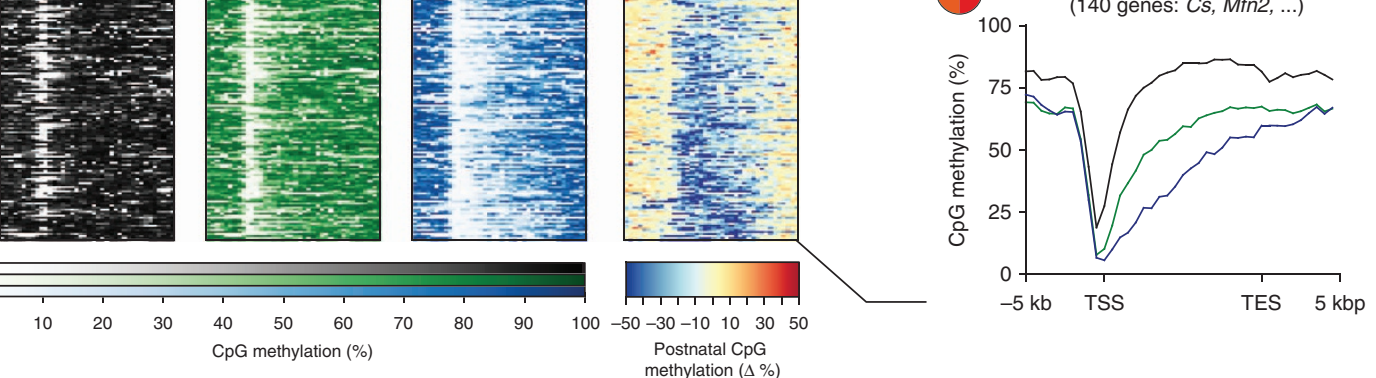

methylation $(\Delta \%)$

Figure 2 | Differentially methylated gene bodies in neonatal and adult cardiomyocytes versus ES cells. (a,b) Heat maps of gene expression (a) and $\mathrm{CpG}$ methylation $(\mathbf{b})$ of gene bodies and flanking regions ( $\pm 5 \mathrm{kbp}$ ) with differential methylation in neonatal and adult cardiomyocytes versus ES cells. Group I, genes with hypermethylated gene bodies in adult cardiomyocytes versus ES cells; group II, genes with hypomethylated gene bodies. (c-f) CpG methylation profiles of grouped genes with selected biological function and flanking regions ( $\pm 5 \mathrm{kbp}$ ). Coloured circles refer to Supplementary Figs 8 and 9. TSS, transcription start site; TES, transcription end site.

cells, was much more prevalent (Fig. 2a,b, group II). This group was highly enriched in genes involved in cardiac transcriptional regulation, cardiac development, muscle contraction and energy supply (Fig. 2d-f; Supplementary Fig. 9). Genes that were further demethylated during postnatal maturation of cardiomyocytes were involved in cardiac contraction or mitochondrial function (Fig. 2e,f).

Genic CpG methylation correlates with gene expression. To gain more insight into the kinetics of DNA methylation, we analysed the time course of gene body demethylation of the
Atp2a2 gene by pyrosequencing (Fig. 3a-f; Supplementary Fig. 4). Demethylation of Atp2a2 in cardiomyocytes was initiated at the $5^{\prime}$ end and progressed towards the $3^{\prime}$ region of the gene (Fig. 3a-d). While the $5^{\prime}$ end of the gene body and upstream TF motifs were already demethylated at birth, the $3^{\prime}$ region lost its CpG methylation by adulthood (Fig. 3b-f). Gene body demethylation correlated with increased expression of Atp2a2 during postnatal cardiomyocyte maturation (Fig. 3g). Adult failing cardiomyocytes expressed lower levels of Atp2a2 than adult control myocytes (Fig. $3 \mathrm{~g}$ ) without significant changes in gene body methylation (Fig. 3b-g). 


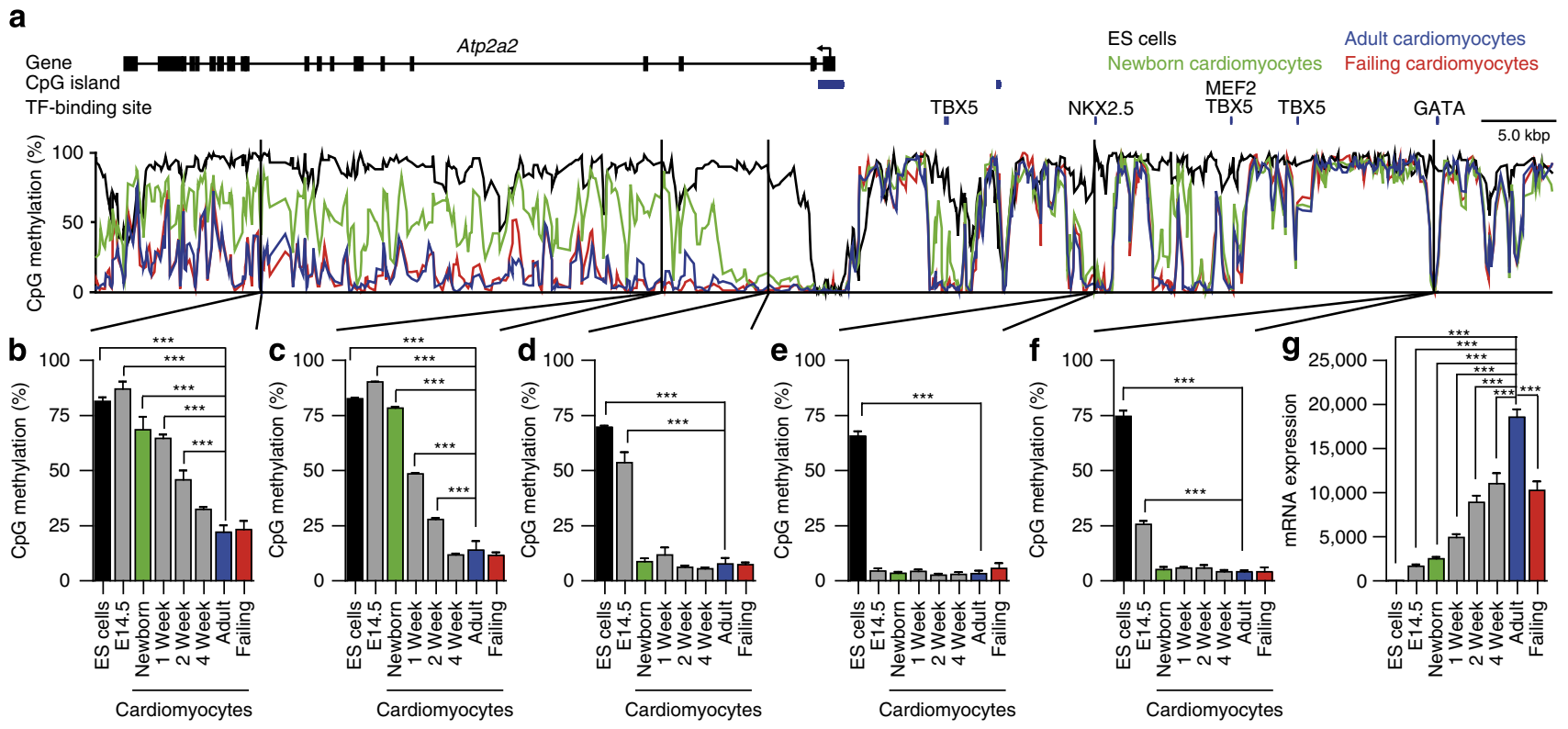

Figure 3 | CpG methylation and gene expression of the Atp2a2 gene. (a-f) Gene body demethylation of the Atp2a2 gene and transcription factorassociated DMRs were assessed during fetal and postnatal development in purified cardiomyocyte nuclei by pyrosequencing (assays I (b), II (c), III (d), V (e), VI (f); Supplementary Fig. 4a). (g) Gene expression of Atp2a2 during fetal and postnatal development (a-g). Data are shown as mean \pm s.e.m., $n=3-9$ per group, ${ }^{\star \star \star} P<0.001$ compared with adult, analysis of variance, Bonferroni post hoc test.

To search for a possible relationship between gene body CpG methylation status and gene expression, we ranked all genes according to their expression level in adult cardiomyocytes and determined the changes in gene expression and DNA methylation after birth (Fig. 4a,b). Those genes that showed the greatest increase in expression after birth (Fig. 4a; expression increase $>250$ fragments per kilobase of transcript per million reads, FPKM) were most strongly demethylated at their gene bodies during postnatal development (Fig. 4b). Genes with demethylation of gene bodies after birth were involved in several metabolic processes (Supplementary Fig. 10). Inactive genes showed a higher level of $\mathrm{CPG}$ methylation at the promoter and the first exon when compared with expressed genes (Fig. 4c). CpG methylation of the first exon and the remainder of the gene was inversely correlated with gene expression (Fig. 4c). Actively expressed genes were decorated with H3K4me1, H3K27ac and H3K4me3 and they lacked H3K27me3 (Fig. 4d-g). In contrast, gene bodies of inactive genes showed abundant $\mathrm{H} 3 \mathrm{~K} 27 \mathrm{me} 3$ in addition to high levels of $\mathrm{CpG}$ methylation (Fig. 4c,g). These genes were grouped into distinct functional categories (Supplementary Fig. 11). Taken together, these data demonstrate that a demethylation wave runs through the gene body of actively transcribed genes. This wave is initiated at the $5^{\prime}$ end of cardiomyocyte genes, becomes further extended until adulthood and correlates with gene expression.

Polycomb-mediated silencing of early developmental genes. The group of genes, which were demethylated in their gene bodies in cardiomyocytes as compared with ES cells (Fig. 2a,b, group II), contained genes that were known to be expressed during cardiac development but showed no significant expression in adult cardiomyocytes. Thus, we asked whether specific histone marks characterize demethylated genes that are inactive in postnatal cardiomyocytes. First, two prototypic genes with either high levels of expression in adult cardiomyocytes (Tnnt2) or expression restricted to early cardiac development (Isl1) were analysed (Fig. 5a,b). Visual inspection of the Tnnt2 locus revealed a demethylated gene body in adult cardiomyocytes, which was covered with active chromatin marks H3K27ac, H3K4me3 and H3K4mel (Fig. 5a). The cardiac differentiation factor Isl1 gene, which is expressed at embryonic day (E) $8.5^{25}$ but not in postnatal cardiomyocytes, remained demethylated until adulthood too. Thus, DNA methylation was not restored on gene inactivation. However, the Isll gene was covered with the repressive chromatin mark H3K27me3 in newborn (Supplementary Fig. 12) and adult cardiomyocytes (Fig. 5b). The gain of this repressive mark set by the enzymatically active subunit enhancer of zeste homologue 2 (EZH2) of the polycomb repressive complex 2 (PRC2) was not restricted to this gene but rather typical for demethylated genes that were not expressed $(<1$ FPKM; Fig. 2a, group II) in adult cardiomyocytes (Fig. 5c, group 1). Affected genes were expressed either in early embryogenesis, such as the transcription factors of the Hox cluster ${ }^{26}$, or at early stages of cardiac development, such as Isl1 and Pitx2 (ref. 27).

We asked whether the affected genes were already marked by H3K27me3 in developmental stages. Analysis of H3K27me3 in newborn cardiomyocytes as well as reanalysis of previously published data sets $^{28}$ were performed. These data revealed enrichment of $\mathrm{H} 3 \mathrm{~K} 27 \mathrm{me} 3$ in embryonic hearts at E12.5 (ref. 28) and in newborn cardiomyocytes (Supplementary Fig. 12a). In addition, EZH2 was found to be localized at these genes in embryonic hearts at E12.5 (ref. 28) (Supplementary Fig. 12a). Recently, the functional importance of PRC2 for proper heart development was shown by cardiac ablation of EZH2 (refs 28,29). Remarkably, a subset of genes including Cdkn2a, Isl1 and the previously described polycomb target genes Six1 and Pax6 (refs 28,29), which were decorated by H3K27me3, showed increased gene expression on EZH2 ablation ${ }^{28,29}$ (Supplementary Fig. 12b-e; Supplementary Table 3). Interestingly, these alterations were more evident in E12.5 hearts as compared with right ventricles of adult hearts ${ }^{28,29}$ (Supplementary Table 3). In contrast, none of the CpG-demethylated and actively transcribed genes ( $>250$ FPKM; Fig. 2a, group II) were marked by $\mathrm{H} 3 \mathrm{~K} 27 \mathrm{me} 3$, but all of these genes were decorated with H3K27ac, H3K4me3 and H3K4me1 (Fig. 5c, group 2). 

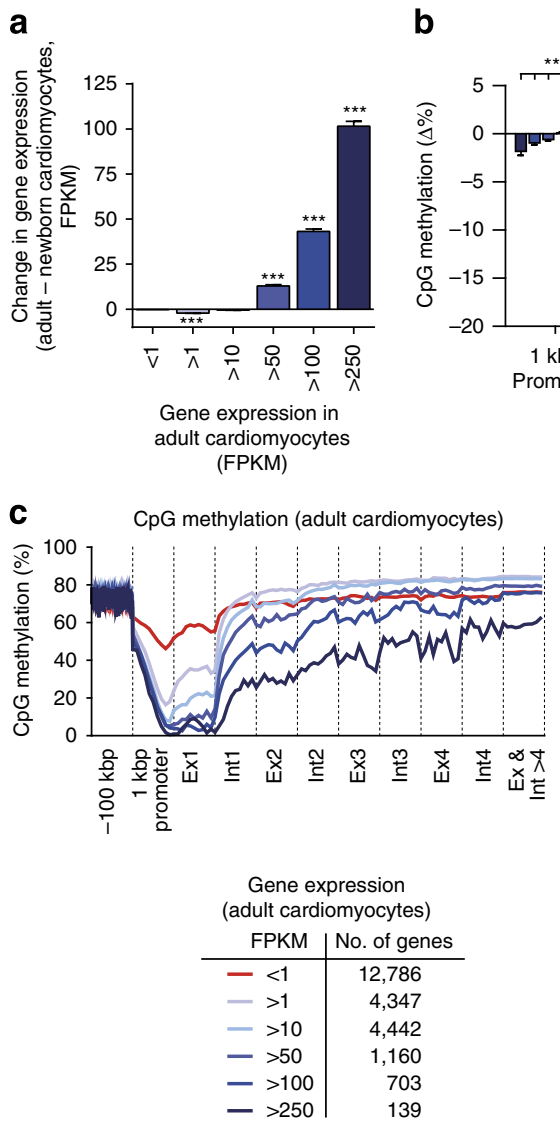

b

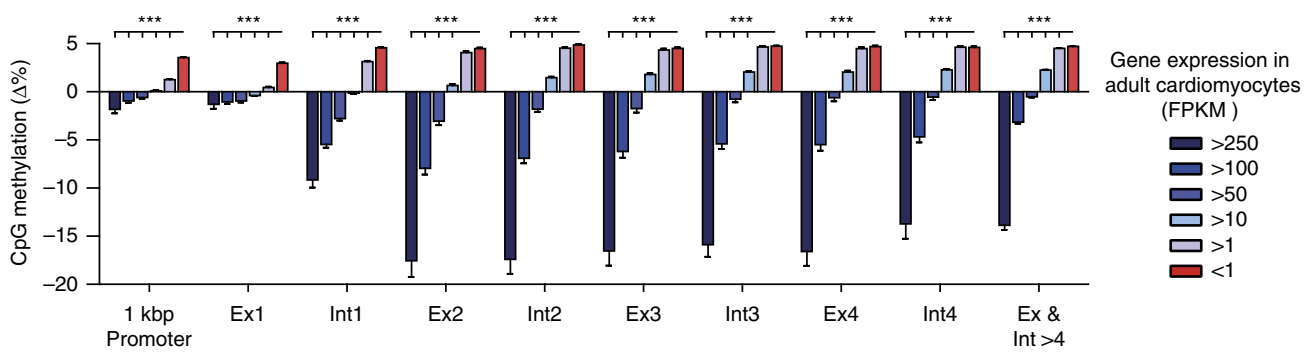

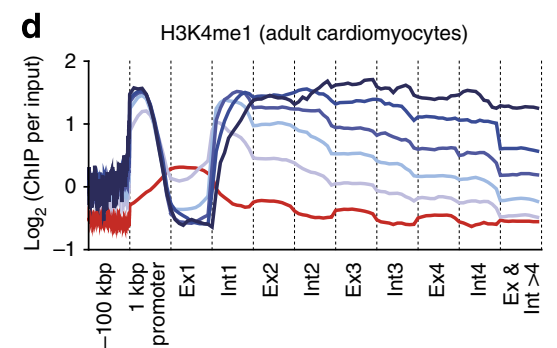

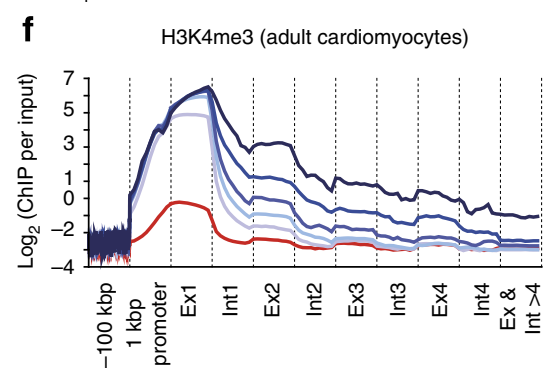

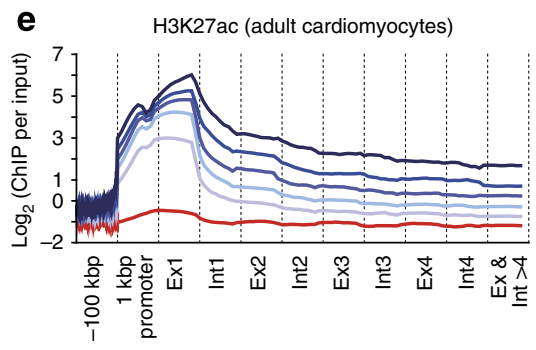

g H3K27me3 (adult cardiomyocytes)

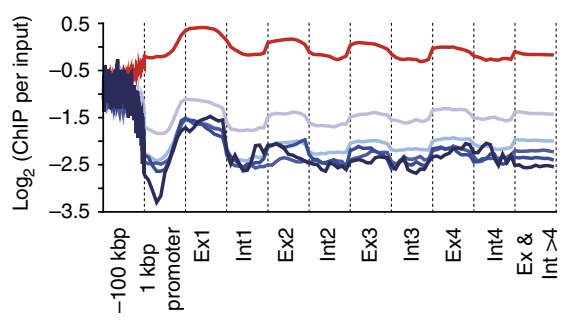

Figure 4 | Correlation of gene expression, CpG methylation and histone modifications in genic regions. (a) Gene expression in adult versus newborn cardiomyocytes was classified according to adult expression levels. Data are shown as mean \pm s.e.m., $n=3$, ${ }^{\star \star \star} P<0.001$ adult versus newborn expression, analysis of variance (ANOVA), Bonferroni post hoc test. (b) Changes in DNA methylation of promoters, exon and intron regions between adult and neonatal cardiomyocytes according to adult gene expression levels. Data are shown as mean \pm s.e.m., $n=3$, ${ }^{\star \star \star} P<0.001$, ANOVA, Bonferroni post hoc test. (c-g) Averaged CpG methylation levels and histone enrichments in genic, promoter ( $1 \mathrm{kbp}$ ) and upstream (100 kbp) regions of adult cardiomyocytes. Genes were grouped according to gene expression levels in adult cardiomyocytes. Ex, exon; Int, intron.

Postnatal de novo DNA methylation requires DNMT3A/B. Postnatal maturation of cardiomyocytes was not only accompanied by demethylation of gene bodies but also by de novo DNA methylation (Supplementary Data 2). Overall, 127 genes gained methylation and 313 lost methylation of their gene bodies during the postnatal period (Fig. 6a). These changes in postnatal DNA methylation of gene bodies correlated inversely with gene expression (Fig. 6a; Supplementary Data 2). Genes that were hypermethylated in cardiomyocytes after birth were involved in myocyte contraction (Fig. 6b), cardiac morphogenesis, cell differentiation and other processes (Supplementary Fig. 11). Functional adaptation of the heart during the postnatal period includes isoform switches in the expression of sarcomere proteins ${ }^{4,5}$. As an example, we analysed DNA methylation and expression of the fetal and adult troponin I isoforms that are encoded by Tnnil and Tnni3 genes, respectively (Fig. 6b-d). The gene body of Tnni1 was demethylated at birth and it was de novo methylated in adult cardiomyocytes (Fig. 6b,c). Postnatal de novo DNA methylation correlated with repression of Tnnil expression (Fig. 6c). In contrast, the adult troponin I isoform Tnni3 was demethylated and turned on in its expression after birth (Fig. 6b,d). To test whether postnatal DNA methylation and gene repression were mediated by de novo DNA methyltransferases 3A/B, mice with cardiac myocyte-specific deletion of the Dnmt3a and Dnmt3b genes were generated and analysed (Fig. 6c,d). Ablation of
DNMT3A/B expression completely prevented postnatal de novo methylation of Tnni1 and partially relieved repression of this gene (Fig. 6c). Similarly, postnatal DNA methylation of the sarcomere components Tpm 2 and the glucose transporter GLUT1 gene (Slc2a1) was mediated by DNMT3A/B (Supplementary Fig. 13). Thus, isoform switching of sarcomere proteins during postnatal life was accompanied by de novo methylation of fetal genes and demethylation of adult isoforms (Fig. 6b).

As postnatal cardiomyocyte maturation is accompanied by cell cycle arrest ${ }^{30,31}$, we analysed postnatal $\mathrm{CpG}$ methylation dynamics of all genes, which were contained in the gene ontology group 'cell cycle' (GO:0007049; Supplementary Fig. 14). Twenty-one out of these 1,279 genes in this group were differentially methylated during postnatal development (Supplementary Fig. 14a). Most of the differentially methylated cell cycle-associated genes showed opposite changes of DNA methylation and RNA expression after birth (Supplementary Fig. 14b) as further illustrated for Ccnd3 and Bmp7 (Supplementary Fig. 14c,d). Both genes showed their highest expression levels at E14.5. Postnatal de novo DNA methylation of $C c n d 3$ and Bmp7 was absent in cardiomyocytes from Dnmt3a/bdeficient mice. However, in these cases, Dnmt $3 a / b$ ablation did not affect gene expression (Supplementary Fig. 14c,d).

Analysis of expression of the group of cell cycle-associated genes revealed that 502 genes were differentially expressed in 
a

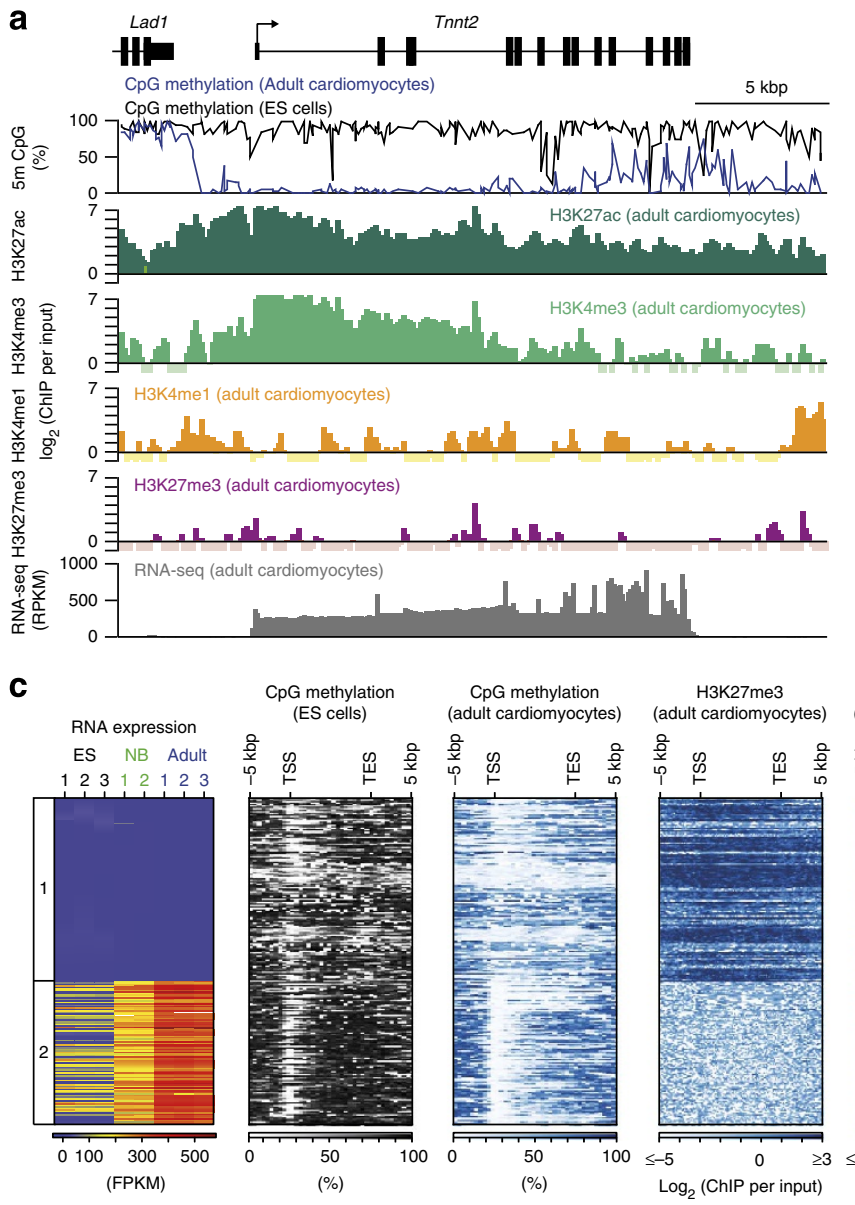

b
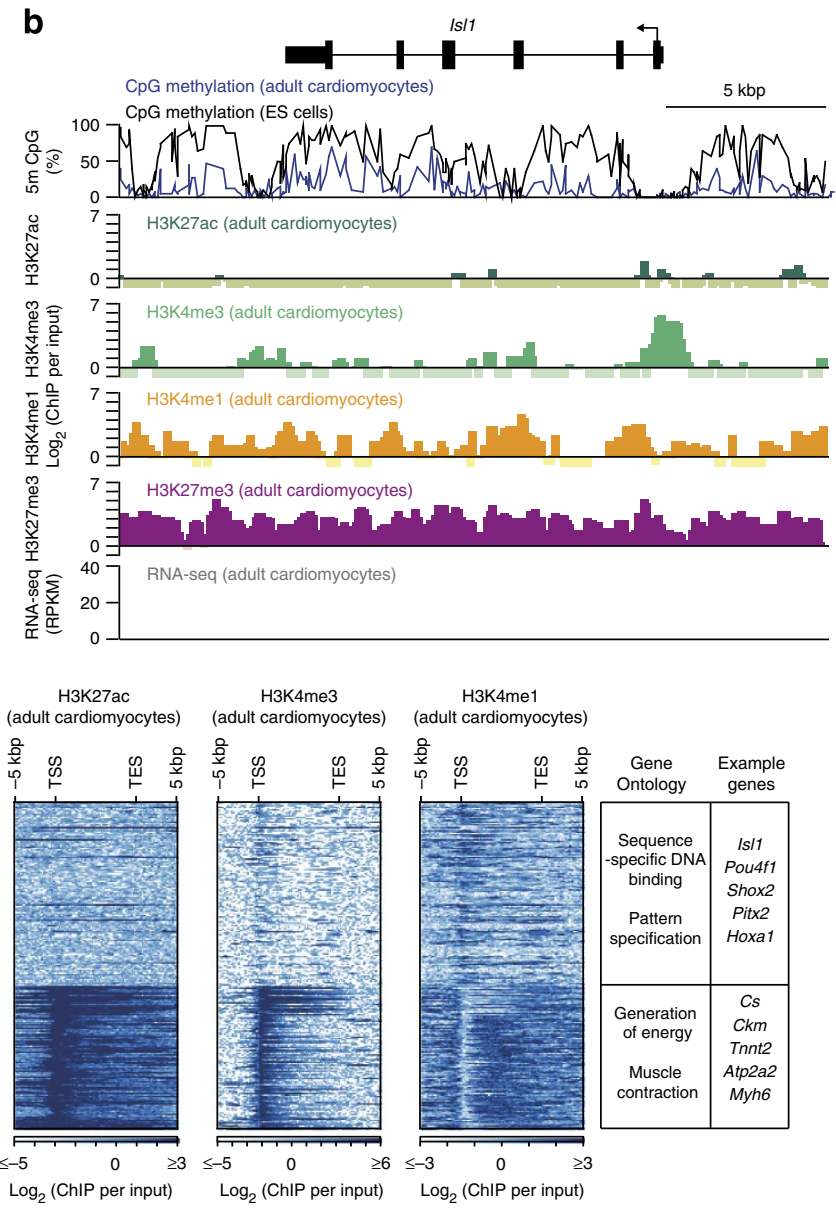

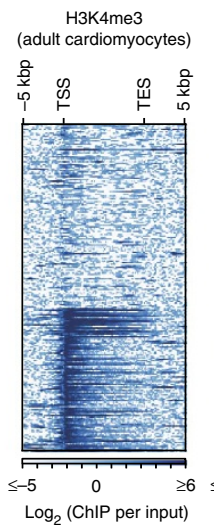

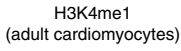

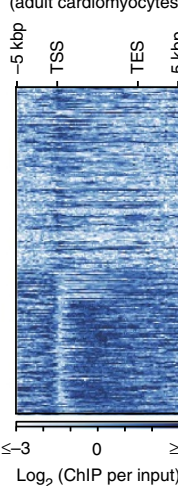

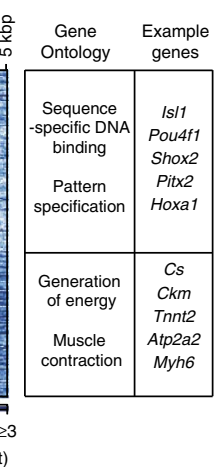

Figure 5 | Repression of genes by the polycomb mark H3K27me3. (a,b) IGV (integrative genomics viewer) traces of CpG methylation of the cardiac troponin T type 2 (Tnnt2) gene (a) and the Is/1 gene (b) in adult cardiomyocytes and ES cells and traces of histone modifications and RNA expression. (c) Heat maps of gene expression, $\mathrm{CpG}$ methylation and histone modifications of genes demethylated in adult cardiomyocytes as compared with ES cells. Displayed genes (derived from Fig. 2a, group II) had either very low ( $<1$ FPKM, group 1) or high gene expression ( $>250$ FPKM, group 2) in adult cardiomyocytes (Supplementary Data 2). Right panel, enriched gene ontology terms $\left(P<10^{-15}\right.$, hypergeometric test, Bonferroni step down correction) and representative genes. TSS, transcription start site; TES, transcription end site; $5 \mathrm{~m} \mathrm{CpG}$, GpG methylation.

adult versus newborn cardiomyocytes (Supplementary Fig. 15a). Thus, we asked whether polycomb-mediated gene silencing is involved in the regulation of these cell cycle genes. However, postnatally repressed cell cycles genes were not decorated with the repressive mark $\mathrm{H} 3 \mathrm{~K} 7 \mathrm{me} 3$ and did not show increased genic $\mathrm{CpG}$ methylation (Supplementary Fig. 15b).

DNA methylation changes after chronic pressure overload. To explore how the cardiac methylome changes in heart disease, we analysed differential $\mathrm{CpG}$ methylation of cardiomyocyte nuclei that were purified from failing murine hearts after chronic left ventricular pressure overload (Supplementary Fig. 16a-e). Compared with developmental alterations, the observed changes in CpG methylation were smaller in scale (Fig. 7a,b). We therefore adjusted the cutoff criteria for DMR detection to $20 \%$ differential methylation. Using these less-stringent criteria, we identified 5,346 disease-associated DMRs (Fig. 7c). These DMRs were mostly intergenic (Fig. 7d). Disease-associated DMRs (5.9\%) showed an overlap with enhancer or promoter regions marked by $\mathrm{H} 3 \mathrm{~K} 27 \mathrm{ac}, \mathrm{H} 3 \mathrm{~K} 4 \mathrm{mel}$ and $\mathrm{H} 3 \mathrm{~K} 4 \mathrm{me} 3$ in adult cardiomyocytes (Fig. 7e). Disease-associated DMRs overlapping with postnatal DMRs were adjacent to genes involved in cardiac muscle cell development, cardiac morphogenesis and energy metabolism (Fig. 7f), indicating adaptation of DNA methylation in disease- relevant regions. Methylation levels of these disease-associated DMRs partially resembled the newborn CpG methylation pattern (Fig. 7g).

\section{Discussion}

The present study resolves the DNA methylome of cardiomyocytes at base-pair resolution from embryonic development to postnatal maturation and disease. For this purpose, cardiomyocyte nuclei were highly purified from frozen heart tissue by FACS and magnetic-assisted sorting. One of the most intriguing features of the cardiomyocyte DNA methylome is a demethylation wave running through gene bodies of cardiomyocyte genes, which orchestrates with de novo DNA methylation and activation of polycomb marks to shape the epigenome of maturing cardiomyocytes after birth (Fig. 8).

Bisulfite sequencing has uncovered distinct patterns of DNA methylation in different genomic contexts, including promoters, gene bodies and regulatory elements ${ }^{12}$. We identified several distinct features of DNA methylation also in cardiomyocytes. When compared with ES cells as a model for an undifferentiated, pluripotent cell type, cardiomyocytes contained short regions of low DNA methylation, which showed characteristics of cisregulatory elements. Tissue-specific DMRs were previously identified for a large number of human and murine cell and 


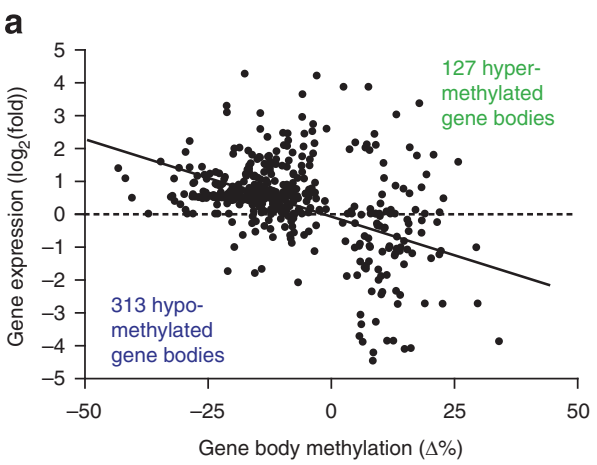

C
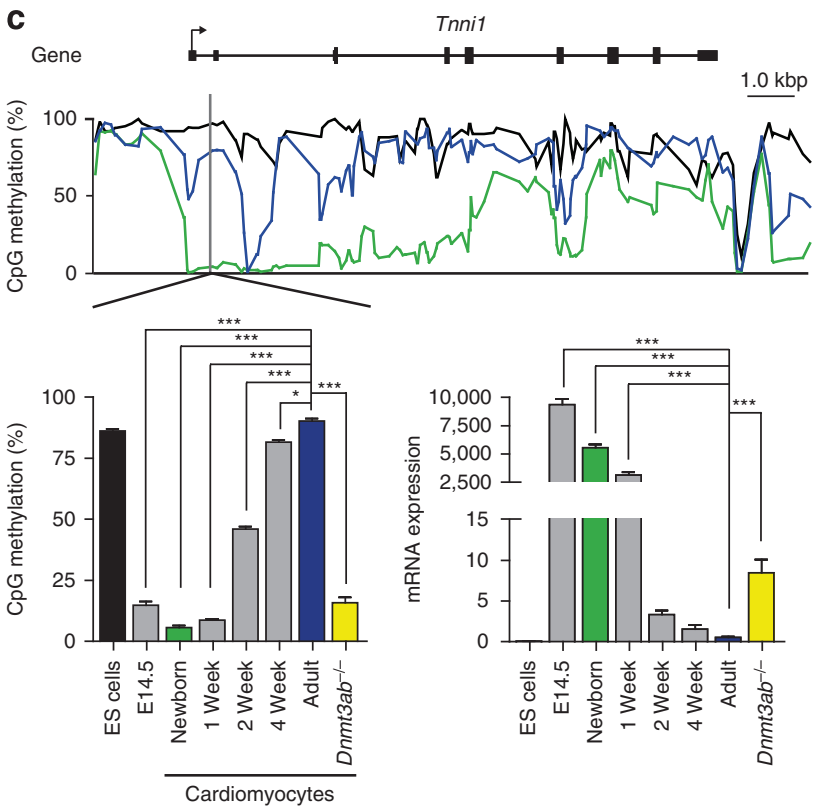
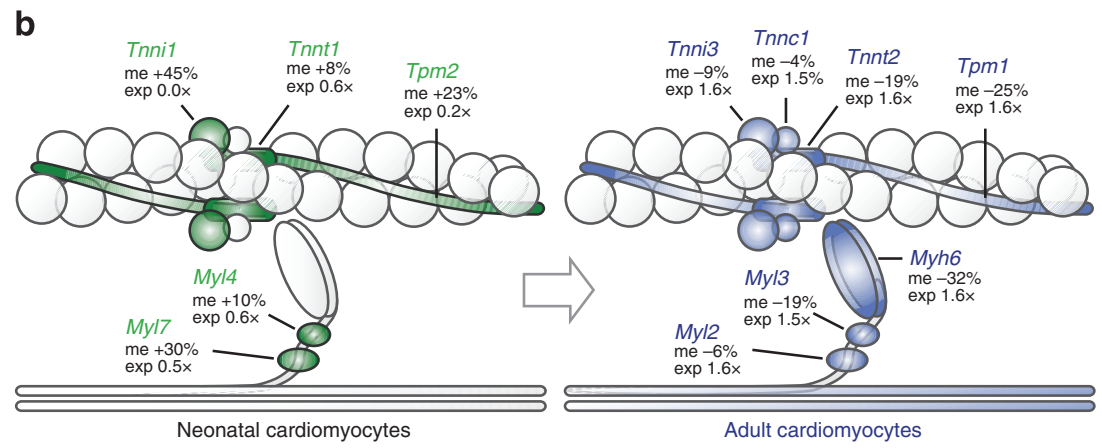

d
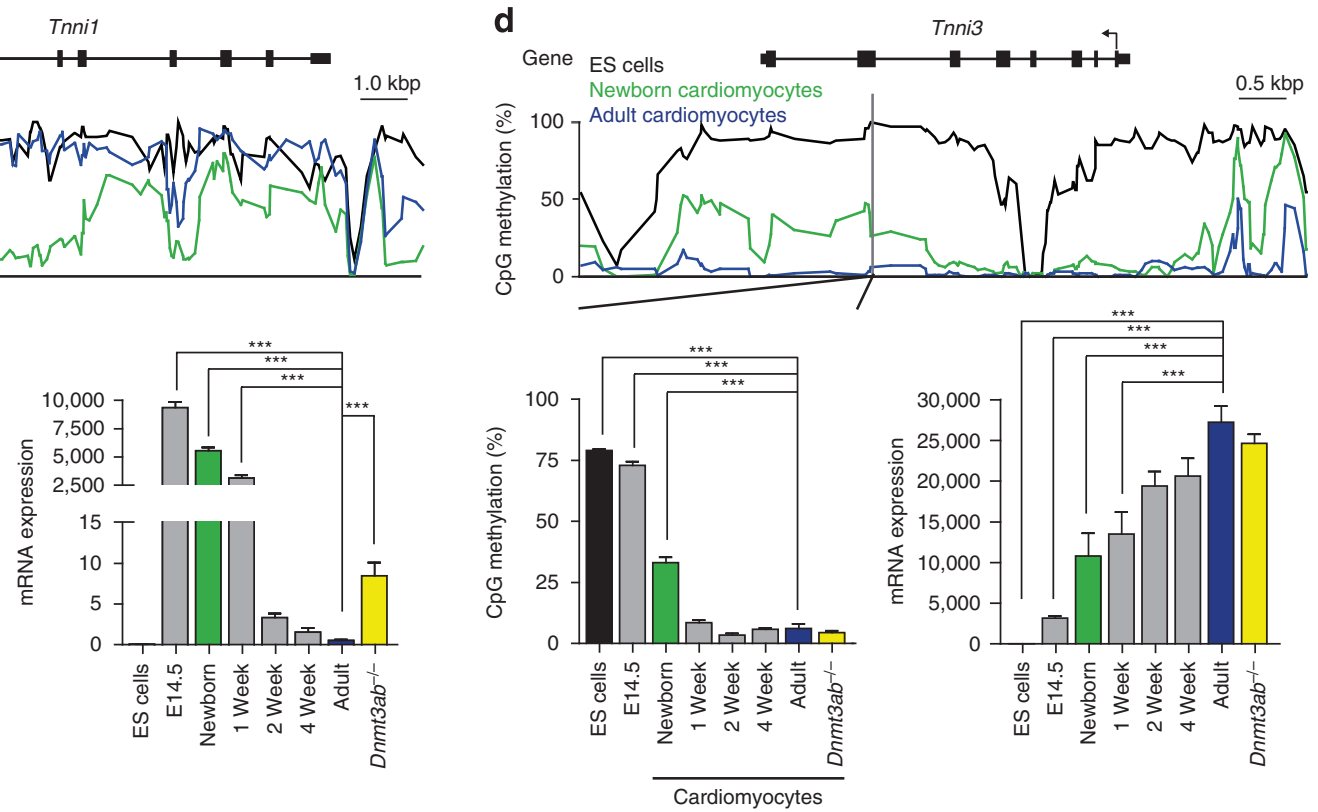

Figure 6 | Repression of genes by de novo DNA methylation. (a) Inverse correlation of DNA methylation changes in cardiac myocytes and gene expression changes during postnatal development $(P<0.0001$, linear regression). (b) Schematic view of a cardiac myofilament at birth (left) and in adult heart (right). Sarcomere components, which are differentially methylated in their genes during postnatal development from birth to adulthood, are filled with green or blue colour. Green colour indicates genes that have low levels of $\mathrm{CpG}$ methylation at birth and are significantly higher methylated in adult cardiomyocytes. Blue colour indicates genes with lower methylation in adult versus neonatal cardiomyocytes. Values next to sarcomere components indicate the difference in gene expression (exp) and $\mathrm{CpG}$ methylation (me) in adult versus newborn cardiomyocytes. (c,d) DNA methylation traces of troponin I genes TnniT (c) and Tnni3 (d) in ES cells, newborn and adult cardiomyocytes (upper panels). Time course of genic DNA methylation (left graphs) and mRNA expression (right graphs). Data are shown as mean \pm s.e.m., $n=3-4$ per group, ${ }^{\star} P<0.05,{ }^{\star \star \star} P<0.001$ compared with adult, analysis of variance, Bonferroni post hoc test; Dnmt3ab ${ }^{-/-}$versus adult control, Student's $t$-test.

tissue types ${ }^{10,22}$. Demethylated regions contained binding motifs for tissue-specific transcription factors and contained histone marks that are characteristic for poised $(\mathrm{H} 3 \mathrm{~K} 4 \mathrm{me} 1)$ or active $(\mathrm{H} 3 \mathrm{~K} 27 \mathrm{ac})$ enhancers ${ }^{10,22}$. Our data demonstrate that demethylation of enhancers was already established in newborn cardiomyocytes and contained cardiac-specific transcription factor motifs that are characteristic for super-enhancers of the cardiac lineage ${ }^{23}$. These cardiomyocyte-specific sites were characterized by $\mathrm{H} 3 \mathrm{~K} 4 \mathrm{me} 1$ marks and part of them also contained $\mathrm{H} 3 \mathrm{~K} 27 \mathrm{ac}$, thus identifying them as poised or active enhancers ${ }^{24}$, respectively. Previous studies have suggested that transcription factor activity could be a key factor for active DNA demethylation of enhancer regions ${ }^{21,32}$. Thus, the DNA methylomes presented in this study provide comprehensive enhancer maps of cardiomyocytes during development and maturation, which extends the previous studies in cardiac tissues.

As a second characteristic feature of the cardiomyocyte DNA methylome, we identified large demethylated regions that covered entire gene bodies of large cardiomyocyte genes, for example, the myosin heavy-chain locus or the Atp2a2 gene (Fig. 8, upper trace). Some of the longest demethylated regions were identified in the genes encoding for the cardiac ryanodine receptor (Ryr2, $180 \mathrm{kbp}$ ), the $\alpha_{1 \mathrm{C}}$-subunit of the L-type $\mathrm{Ca}^{2+}$ channel (Cacna1c, $162 \mathrm{kbp}$ ) or titin (Ttn, $121 \mathrm{kbp}$; Supplementary Data 2). The significance of DNA methylation in gene bodies is still only partially understood ${ }^{12}$ and studies have shown both positive or negative correlations between genic DNA methylation and gene expression. In neurons ${ }^{33}$, hematopoietic stem cells ${ }^{34}$ and other lineages ${ }^{35}$, demethylation of regions containing the transcription start and gene bodies was identified in highly expressed genes. In contrast, a positive correlation between CpG methylation and gene expression was identified in human ES cells ${ }^{36}$, mouse oocytes $^{37}$ or B lymphocytes ${ }^{38}$. In the present study, gene bodies were demethylated starting at the $5^{\prime}$ end and extending towards the $3^{\prime}$ region during postnatal maturation of cardiomyocytes. Genic demethylation correlated well with active histone marks and increased expression of cardiomyocyte genes. 

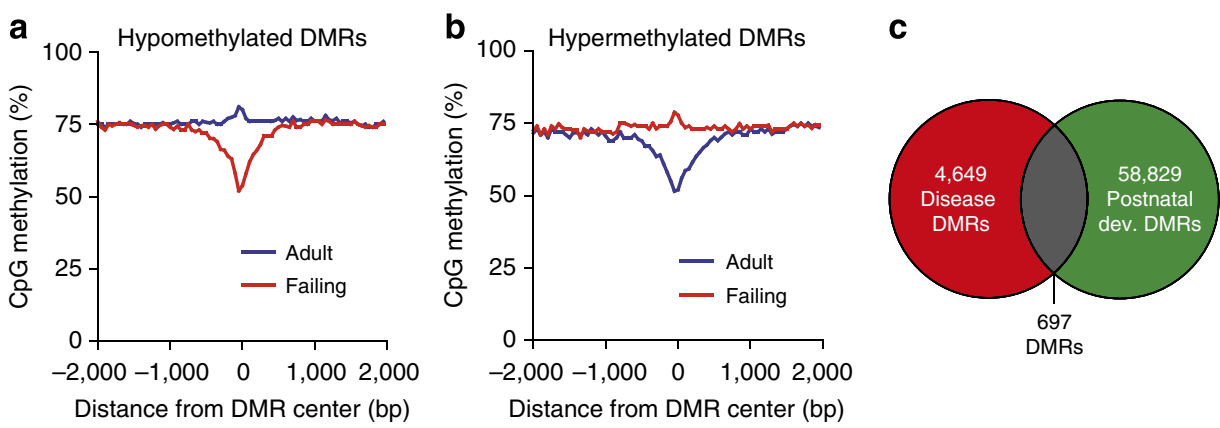

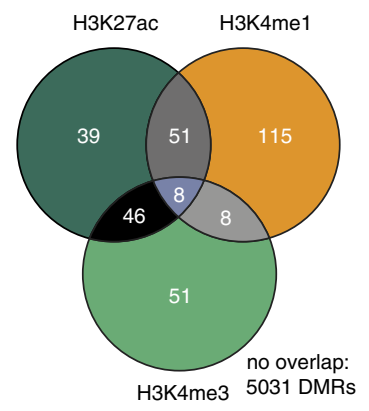

f

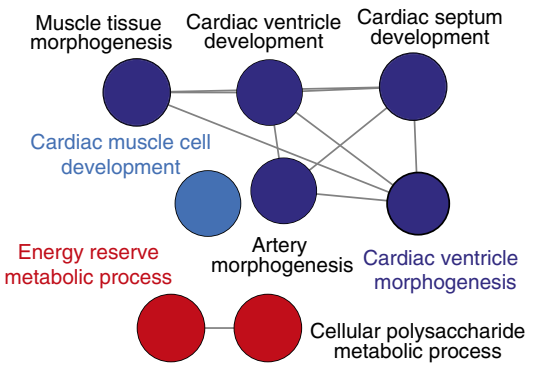

g

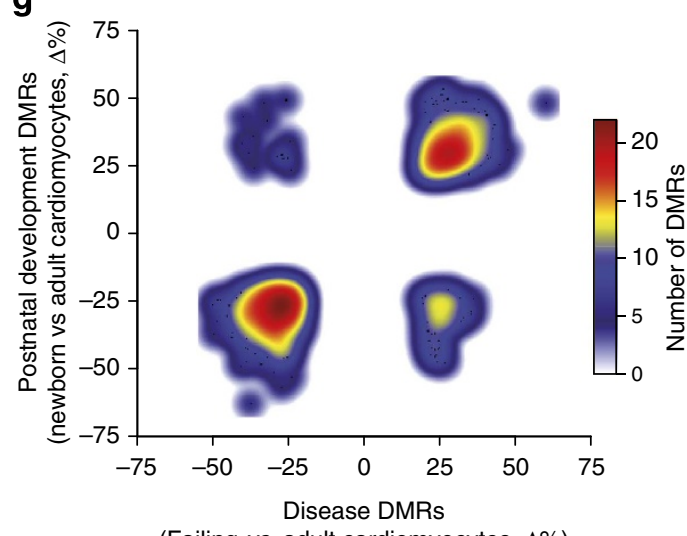

(Failing vs. adult cardiomyocytes, $\Delta \%$ )

Figure 7 | Developmental pattern of CpG methylation in cardiomyocytes in experimental murine heart failure. (a,b) Overlay plot of $\mathrm{CpG}$ methylation in DMRs and surrounding regions of cardiomyocytes isolated from healthy or failing adult hearts displaying hypomethylated (a) and hypermethylated (b) DMRs. (c) Venn diagram showing partial overlap ('common DMRs') of disease DMRs (differential methylation between failing and healthy cardiomyocytes) and postnatal DMRs (differential methylation between healthy newborn and adult cardiomyocytes). (d) Genome annotation of DNA regions with differential $\mathrm{CpG}$ methylation between failing and healthy adult cardiomyocytes. (e) Intersection of disease DMRs with $\mathrm{H} 3 \mathrm{~K} 27 \mathrm{ac}$, $\mathrm{H} 3 \mathrm{~K} 4 \mathrm{me} 1$ and H3K4me3 peaks in adult cardiomyocytes. (f) Functional annotation of common DMRs in postnatal development and disease (hypergeometric test, Bonferroni step down correction, $P$ value per $G O$ term $<0.02)$. (g) Density plot of common DMRs showing direction of methylation changes in postnatal development and failure. TTS, transcription termination site (region $-100 \mathrm{bp}$ to $+1 \mathrm{kbp}$ ); UTR, untranslated region.

However, not all genes that were demethylated in cardiomyocytes versus ES cells were also expressed in postnatal cardiomyocytes. More than one hundred genes were DNA demethylated and decorated by trimethylation of $\mathrm{H} 3 \mathrm{~K} 27$ (Fig. 8, middle trace). This histone mark is associated with polycomb-mediated gene repression, and EZH2 is one of the core proteins of the PRC2 in the heart ${ }^{28,29}$. Deficiency of EZH2 in cardiomyocytes caused lethal heart malformations ${ }^{28}$ or postnatal cardiomyopathy ${ }^{29}$, depending on the time of EZH2 ablation. Binding of EZH2 and $\mathrm{H} 3 \mathrm{~K} 27 \mathrm{me} 3$ could be identifed at E12.5 and H3K27me3 marks persisted at the same loci in newborn and adult cardiomyocytes. Demethylated genes that were transiently expressed during embryonic development, for example, Isl1 (ref. 25) and Pitx2 (ref. 27), were stably repressed by H3K27me3. However, deletion of Ezh2 led to reactivation of Isl1, Pitx2 and other developmental genes ${ }^{28,29}$. It is interesting to note that H3K27me3 was specifically associated with repressed and demethylated genes in cardiomyocytes. This finding is supported by previous studies that have detected H3K27me3mediated gene repression in genomic regions lacking DNA methylation 34,35 . Most recently, it was shown that polycomb may selectively be recruited to unmethylated DNA sequences and transcriptional activity or CpG methylation prevented polycomb binding and function ${ }^{39}$.

As a second mechanism for repression of demethylated cardiomyocyte genes, we identified de novo $\mathrm{CpG}$ methylation mediated by DNA methyltransferases 3A/B (Fig. 8, lower trace).
Genes that were hypo- or hypermethylated in cardiomyocytes after birth were involved in multiple physiological processes including the transition from fetal to adult sarcomeric gene expression. To adapt to the specific demands of pre- versus postnatal life, several protein isoforms of the sarcomere are switched during the perinatal phase by an unknown mechanism ${ }^{5}$. Indeed, we found the slow skeletal troponin I isoform (Tnni1) to be repressed by postnatal DNA methylation, which was mediated by DNMT3A/B. Cardiomyocyte-specific ablation of DNMT3A/B completely prevented Tnnil methylation after birth and partially reactivated gene expression. The perinatal switch from fetal to adult sarcomere protein isoforms has been associated with important aspects of myocyte development and contractile function ${ }^{4,5}$. In particular, the decrease in $\mathrm{Ca}^{2+}$ sensitivity of the sarcomere has been linked with the transition from slow skeletal (Tnni1) to cardiac troponin I (Tnni3) ${ }^{4}$. In addition to the fetal sarcomere isoforms, genes involved in the fetal to adult transition of energy metabolism ${ }^{3}$ and several other process were differentially methylated in their gene body after birth. These findings suggest that dynamic gene methylation may play an important role in cardiomyocyte switching and maturation after birth.

To determine possible alterations in the DNA methylome during cardiac disease, we have subjected mice to 3 weeks of cardiac pressure overload. Constriction of the aortic arch resulted in ventricular hypertrophy, interstitial fibrosis and pulmonary oedema, thus partially resembling the phenotype of chronic 


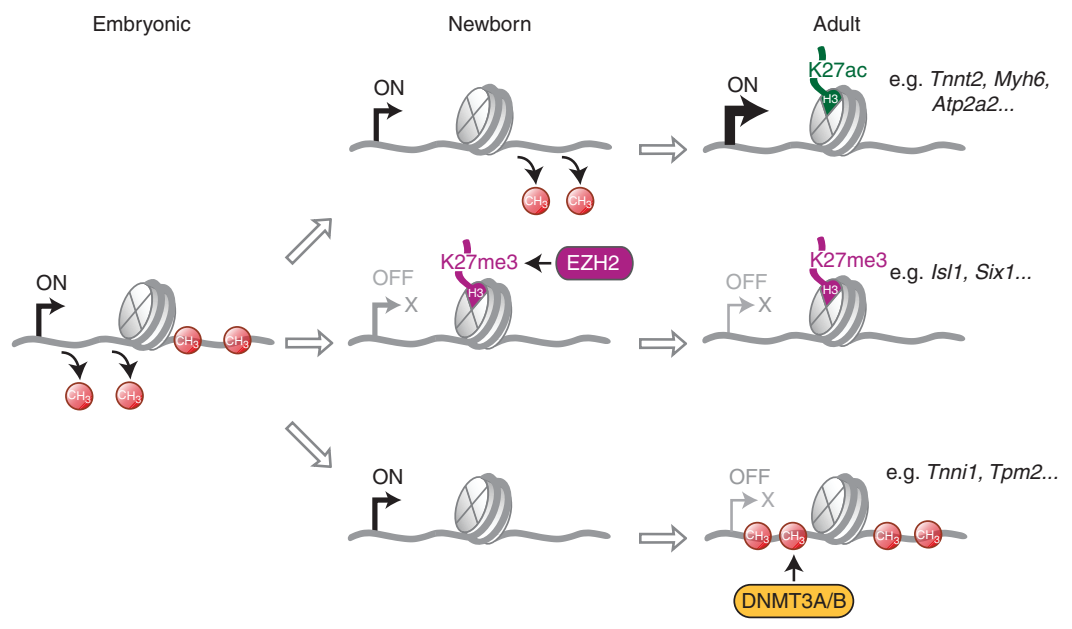

Figure 8 | Dynamics of DNA methylation and histone modification in genes bodies of cardiomyocyte genes during development. Gene bodies of cardiomyocyte genes are sequentially demethylated from embryonic stages (left part, E14.5, data from Fig. 3) until newborn (middle part) and adult stages (right part). The degree of gene body demethylation correlates with histone H3K27ac marks and high expression levels of cardiomyocyte genes (upper trace, data from Figs 1, 3, 5 and 6; Supplementary Fig. 4). Demethylated genes, which are transiently expressed during embryonic development, can be repressed by EZH2-mediated H3K27 trimethylation (middle trace, data from Fig. 5; Supplementary Fig. 12). Demethylated genes may also be repressed by de novo methylation, which is mediated by DNMT3A/B (lower trace, data from Fig. 6; Supplementary Fig. 14).

human heart failure. While DNA methylation in failing cardiomyocytes returned to a fetal methylation pattern, the observed changes were less promiment and abundant compared with physiological postnatal myocyte maturation. Thus, future studies will be important to determine whether the human cardiomyocyte DNA methylome shows similar features during development and in disease. Recent studies have investigated DNA methylation in human cardiac tissue biopsies from patients with chronic heart failure of different etiologies ${ }^{17,18}$. Both studies described distinct signatures of DNA methylation in failing versus non-failing hearts. The present study describes a path to assign such alterations to a specific cell type in the heart. Future studies will be important to uncover the DNA methylomes of other cardiac cell types, for example, fibroblasts, endothelial cells, immune cells and so on, to determine their epigenetic contribution to cardiac development and disease.

The present study establishes DNA methylation in cardiomyocytes as dynamic and reversible during development, postnatal maturation and disease. Future studies unravelling the signals and mechanisms involved in shaping the cardiac DNA methylome will be essential for a better understanding of cardiac myocyte biology.

\section{Methods}

Animal procedures. All animal procedures were approved by the responsible animal care committee (Regierungspräsidium Freiburg, Germany) and they conformed to the Guide for the Care and Use of Laboratory Animals published by the National Academy of Sciences, 2011. Mice with cardiac myocyte-specific ablation of DNMT3A/B expression were generated by crossing Dnmt3aflox and Dnmt3b flox mice ${ }^{40}$ with mice expressing cre recombinase under control of the cardiac Mlc2a promoter ${ }^{41}$. Male mice of the indicated ages were dissected to obtain cardiac tissues. To induce cardiac pressure overload by transverse aortic constriction for 3 weeks, male mice ( 8 weeks old) were anaesthetized with $2 \%$ (vol) isoflurane in oxygen. After thoracotomy, a 7.0 silk suture was placed around a $27-\mathrm{G}$ hypodermic needle to constrict the transverse aorta ${ }^{42}$.

ES cells. Murine ES cells, which were originally derived from blastocysts of mixed 129-C57BL6 background ${ }^{21}$, were maintained in ES cell medium (DMEM, 15\% fetal calf serum (FCS), non-essential amino acids, $10 \mathrm{ng} \mathrm{ml}^{-1}$ leukaemia inhibiting factor, $0.1 \% \beta$-mercaptoethanol) on gelatine-coated plates $^{21}$.

Echocardiography. Echocardiography was performed using a Vivid 7 Dimension (GE Healthcare, Munich, Germany) echocardiograph equipped with a $14-\mathrm{MHz}$ transducer ${ }^{42}$. Parasternal short-axis views were used for M-mode analysis. Enddiastolic and endsystolic left ventricular inner diameters (LVIDd, LVIDs) were measured and fractional shortening (FS) and ejection fraction (EF) were calculated using the following equations: $\mathrm{FS}=(\mathrm{LVIDd}-\mathrm{LVIDs}) / \mathrm{LVID}, \mathrm{EF}=\left(\mathrm{LVIDd}^{3}\right.$ - LVIDs $^{3}$ )/LVIDd ${ }^{3}$ (ref. 42)

Histology. Hearts were fixed with $4 \%$ paraformaldehyde in phosphate-buffered saline (PBS), embedded in paraffin, cut into $3 \mu \mathrm{m}$ slices and stained with haematoxylin-eosin, Sirius-red or fluorescent wheat germ agglutinin (WGA; Alexa Fluor 488 conjugate, Invitrogen, Karlsruhe, Germany). Nuclei were counterstained with propidium iodide (Sigma, Munich, Germany). For immunohistochemical staining, hearts were snap frozen, embedded in optimum cutting temperature (OCT) medium (Tissue-Tek), cut into $3 \mu \mathrm{m}$ slices and stained with an antibody against PCM1 (1:1,000, HPA023370, Sigma) in combination with a Cy3-labelled anti-rabbit antibody (1:1,000, Invitrogen). Glycocalyx was stained with WGA (Alexa Fluor 488 conjugate, Invitrogen) and nuclei were counterstained with DAPI (4',6-diamidino-2-phenylindole, Invitrogen).

Flow cytometric analysis and sorting of cardiomyocyte nuclei. Cardiomyocyte nuclei were isolated from cardiac tissue ${ }^{20}$ and were stained by an antibody directed against PCM1 (1:1,000, HPA023370, Sigma) and an Alexa488-labelled secondary anti-rabbit antibody (1:1,000, Invitrogen) or a molecular beacon targeting $\mathrm{mRNA}^{43}$ of Tnnt2 (Supplementary Data 3; $50 \mathrm{nM}$ ) for $30 \mathrm{~min}$. Nuclei were identified by 7-aminoactinomycin D (7-AAD) (1:500, Invitrogen). For analysis of $\alpha \mathrm{MHC}-\mathrm{H} 2 \mathrm{~B}-$ mCherry transgenic mice, an Alexa647-labelled secondary anti-rabbit antibody (1:1,000, Invitrogen) and for nuclei-staining DAPI (1:1,000, Invitrogen) was used. Nuclei were analysed or sorted by flow cytometry (CyFlow Space, Partec, Münster, Germany; BD Influx cell sorter, BD Biosciences, Heidelberg, Germany; Bio-Rad S3 cell sorter, Bio-Rad Laboratories, Munich, Germany).

Magnetic-assisted sorting. Magnetic-assisted cell sorting (MACS) of myocyte nuclei was performed after isolation and staining of nuclei with a PCM1 antibody (1:1,000, HPA023370, Sigma). Nuclei were centrifuged and resuspended in $80 \mu \mathrm{l}$ MACS buffer. Anti-rabbit IgG MicroBeads ( $20 \mu$; Miltenyi, Bergisch Gladbach, Germany) were added and incubated for $15 \mathrm{~min}$ at $4{ }^{\circ} \mathrm{C}$. MACS buffer $(900 \mu \mathrm{l})$ was added and the nuclei suspension was applied to an M column (Miltenyi), washed with $3 \mathrm{ml}$ MACS buffer and eluted with $1 \mathrm{ml}$ MACS buffer after removal of the magnet. The eluate was applied to another M column, washed again and eluted in $1 \mathrm{ml}$ elution buffer (1 mM EDTA in PBS). Sorting efficiency was determined by staining aliquots before and after cell separation with an Alexa488-labelled secondary anti-rabbit antibody (1:1,000, Invitrogen) and by flow cytometric analysis (CyFlow Space, Partec).

Isolation of cardiomyocytes for transcriptome analysis. Adult and neonatal hearts were dissociated to obtain a single-cell suspension ${ }^{44,45}$. Adult hearts were dissociated by retrograde perfusion with Tyrode's solution supplemented with $25 \mathrm{mM}$ butanedione monoxime, $2 \mathrm{mM} \mathrm{CaCl}_{2}, 0.8 \mathrm{mg} \mathrm{ml}^{-1}$ collagenase B (Roche, Mannheim, Germany), $0.4 \mathrm{mg} \mathrm{ml}^{-1}$ hyaluronidase (Sigma) and $3 \mu \mathrm{g} \mathrm{ml}^{-1}$ trypsin (Sigma) for $12 \mathrm{~min}$. Hearts were gently dissected and enzymatic digestion was 
stopped by addition of Tyrode's buffer supplemented with 5\% FCS, $25 \mathrm{mM}$ butanedione monoxime and $2 \mathrm{mM} \mathrm{CaCl}$. Newborn hearts were dissociated by several rounds of digestion with trypsin (Gibco) in Hank's Balanced Salt Solution (HBSS, Life Technologies). Digestion was stopped by resuspension in HBSS containing $4 \%$ FCS. Cell suspensions of adult and neonatal hearts were kept at $4{ }^{\circ} \mathrm{C}$ until sorting. Sorting of cardiomyocytes from cardiac cell suspensions was carried out at $4{ }^{\circ} \mathrm{C}$ using a Bio-Rad S3 cell sorter. Cardiomyocytes and non-cardiomyocytes were visualized using the cell-permeable nucleid acid stain Vybrant DyeCycle Ruby (Life Technologies). Non-viable cells were identified by counterstaining with the cell-impermeable nucleid acid stains Sytox Green or 7-AAD (Life Technologies). Cardiomyocytes were discriminated from other cardiac cells by high forward scatter (FSC) signals. FSC pulse width was used to exclude doublets from sorting. Gates were selected to sort viable cardiomyocytes directly into RLT lysis buffer (Qiagen, Hilden, Germany). To confirm the gating strategy, small aliquots were permeabilized with $0.1 \%$ saponin (Sigma) and stained with antibodies against cardiac troponin I (ab47003, Abcam) and $\alpha$-actinin (At7811, Sigma) in combination with secondary Alexa Fluor-coupled antibodies (Life Technologies).

External data sets. Previously published MethylC-seq data from murine ES cells $(\text { GSE30206) })^{21}$ and murine adult heart (GSM1051154) ${ }^{10}$ were downloaded, mapped and analysed in the same way as the methylomes generated in this study. Previously published chromatin immunoprecipitation-sequencing (ChIP-seq) data for H3K27ac (GSM851290) and H3K4mel (GSM851287) for embryonic heart $\left(\right.$ E14.5) ${ }^{46}$ and ES cells (GSM594577 and GSM594579) ${ }^{24}$ were reanalysed. ChIP-seq data for H3K27me3 and EZH2 for E12.5 hearts (GSE29994) were reanalyzed ${ }^{28}$. RNA-seq data of $E z h 2$ mutant mice and corresponding controls were reanalyzed $(\text { GSE29992) })^{28,29}$

Whole-genome bisulfite sequencing. MethylC-seq was performed as described $^{36}$. In brief, $1 \mu \mathrm{g}$ of genomic DNA was extracted from purified nuclei (AllPrep DNA/RNA Mini Kit, Qiagen). DNA-seq libraries were prepared with methylation adapters (ILMN ME-100-0010, Illumina, San Diego, USA), bisulfite conversion was carried out with the EZ DNA methylation kit (D5001, Zymo Research, Freiburg, Germany) for $16 \mathrm{~h}$ and final PCR was performed with the KAPA Uracil + system (16 cycles, KAPAbiosystems KK2801). All libraries were sequenced on a paired-end 101-bp sequencing run (Illumina HiSeq 2000).

CpG methylation analysis. All bioinformatic tools used in this study were integrated into the Galaxy web server ${ }^{47-49}$. The generated data format is compliant with the BED file format. Sequencing reads were trimmed (Phred quality score \pm 20 ) and mapped using Bismark ${ }^{50}$ (bowtie 1 (ref. 51), mouse genome assembly $\mathrm{mm} 9$ ) and PCR duplicates were removed with SAMtools ${ }^{52}$. For further analysis, we developed a set of bioinformatic tools (Methtools; https://github.com/ bgruening/methtools), including differential methylation analysis. Results for the sense and antisense strand were combined after calling of $\mathrm{CpG}$ methylation values. Only CpGs with a minimal coverage of 4 were included in further analysis steps. In case of ES cell data ${ }^{21}$, SNPs between C57BL/6J and 129S5 mouse strains were filtered. Methylation values were smoothed using a running average of three consecutive CpGs. For determination of DMRs of two methylomes (DMRs), data sets were intersected to exclude CpGs determined in only one data set using Bedtools $^{53}$. A sliding window approach was used to identify DMRs. Regions displaying a mean CpG methylation delta $\geq 40 \%$ over five CpGs between methylomes were selected as DMRs. Additional exclusion criteria were a minimal difference of $10 \%$ for each individual CpG and a maximal distance of $1,000 \mathrm{bp}$ between adjacent CpGs. Selected regions were extended stepwise until they failed to fulfil the cutoff criteria and were then reported as DMRs. In case of diseaseassociated DMRs, a mean delta of $20 \%$ or greater was accepted. Differential gene body methylation between ES cells and adult cardiomyocytes was identified by DMRs covering $>25 \%$ of a gene body with a minimal DMR length of $1 \mathrm{kbp}$. For comparison of newborn and adult cardiomyocytes, the cutoff criterion was $>5 \%$ of the gene body. Genes smaller than $500 \mathrm{bp}$ were excluded from all analyses. The Integrative Genomics Viewer ${ }^{54}$, Bioconductor ${ }^{55}$ and deepTools ${ }^{56}$ were used for visualization.

Motif analysis and binding site prediction. Location of transcription factorbinding sites and motif enrichments within DMRs were determined by the Homer tool $^{57}$ using default parameters. The known motifs used in this study were derived from the Homer tool and were validated by ChIP-seq. Motifs lacking this experimental proof were omitted. HAND1/HAND2 motifs were derived from JASPAR.

Pyrosequencing. Genomic DNA (500 ng) was extracted from MACS- or FACS-purified nuclei (AllPrep DNA/RNA Mini Kit, Qiagen) and was bisulfite converted with the EZ DNA Methylation Kit (D5001, Zymo Research). The concentration of converted DNA was adjusted to $5 \mathrm{ng}^{-1} \mathrm{l}^{-1}$ and DNA was amplified with the PyroMark PCR Kit (Qiagen) using a three-primer approach with a universal biotinylated primer ${ }^{58}$. PCR products were checked by gel electrophoresis, pyrosequenced using PyroMark Gold Q24 Reagents (Qiagen) on a PyroMark Q24 instrument ${ }^{59}$ and quantified with the PyroMark Q24 software (Qiagen). For each assay, a standard curve of $0,25,50,75$ and $100 \%$ methylated DNA was measured. The GenomePlex Complete WGA Kit (Sigma) was used to generate unmethylated DNA and the CpG methyltransferase M.Sssl (NEB, Frankfurt, Germany) was used to generate methylated DNA. Primer sequences are listed in Supplementary Data 3.

Annotation of cis-regulatory regions. Prediction of functional significance of regions was performed using the GREAT tool ${ }^{60}$. The basal gene regulatory domain was defined as the region starting $5 \mathrm{kbp}$ upstream and ending $1 \mathrm{kbp}$ downstream of the TSS. The gene regulatory domain was extended in both directions up to $50 \mathrm{kbp}$ to the nearest gene's basal domain.

Gene ontology analysis. ClueGO ${ }^{61}$ was used to find over-represented GO terms in the categories 'biological process', 'cellular component' and 'molecular function'. Benjamini-Hochberg correction was performed for multiple testing-controlled $P$ values. Significantly enriched terms were functionally grouped and visualized. The GO term connectivity threshold was adjusted to $>0.3$ (kappa value). The highest significant term of the group was displayed as leading term.

CpG island and genomic annotation. Random sequences from MethylCseq-assessable genomic regions were selected to analyse genome representation of genomic features and $\mathrm{CpG}$ islands. Genomic feature annotation was performed using Homer tools ${ }^{57}$. CpG island annotation ${ }^{62}$ and UCSC gene prediction annotation ${ }^{63}$ were downloaded from UCSC.

Gene expression analysis. Polyadenylated RNA was isolated from $500 \mathrm{ng}$ of total RNA with magnetic beads (NEBNext Poly(A) mRNA Magnetic Isolation Module, NEB). Libraries were constructed (NEBNext Ultra RNA Library Prep Kit for Illumina, NEB) and size selection was performed with AMPure XP Beads (Beckman Coulter, Krefeld, Germany). Thirteen PCR cycles were used for library amplification and were sequenced on a HiSeq 2500 (50 bp, Illumina, San Diego, USA). RNA-seq data were mapped to the $\mathrm{mm} 9$ genome assembly using Tophat ${ }^{64}$. FPKM values were calculated by Cufflinks ${ }^{65}$ as an estimate of transcript expression. Differential gene expression was calculated using Cuffdiff ${ }^{65}$. The analysis was performed with default parameters and the UCSC gene annotation ${ }^{63}$ as a reference. Quantitative real-time PCR was carried out as described ${ }^{66}$. Primer sequences are listed in Supplementary Data 3.

ChIP-sequencing. ChIP-seq was performed as described with modifications ${ }^{67}$. Lysis buffers for preparation of nuclei were supplemented with $10 \mathrm{mM}$ sodium butyrate (Sigma). MACS-purified nuclei were fixed with $1 \%$ freshly prepared paraformaldehyde (Sigma) for $2 \mathrm{~min}$ at room temperature. Fixation was stopped by adding glycine to a final concentration of $125 \mathrm{mM}$. Nuclei were washed three times with PBS and were lysed in SDS buffer (50 mM Tris- $\mathrm{HCl}$, pH 8.0, $10 \mathrm{mM}$ EDTA, $1 \%$ SDS) supplemented with protease inhibitors (Roche Complete Protease Inhibitor Cocktail, Roche). Chromatin was sheared using a Bioruptor (Diagenode, Liege, Belgium) with 30 high-energy cycles ( $30 \mathrm{~s} \mathrm{on} / 30 \mathrm{~s}$ off) to an average size of 200-400 bp. Lysates were diluted 1:10 with buffer ( $22 \mathrm{mM}$ Tris-HCl, $\mathrm{pH} 8.0$, $165 \mathrm{mM} \mathrm{NaCl}, 2.2 \mathrm{mM}$ EDTA, $1.1 \%$ Triton X-100) and were precleared with $0.1 \%$ bovine serum albumin for $2 \mathrm{~h}$ at $4{ }^{\circ} \mathrm{C}$. Antibodies were preincubated with Protein A Dynabeads (Invitrogen) for $2 \mathrm{~h}$ at $4{ }^{\circ} \mathrm{C}$. For immunoprecipitation, either $5 \mu \mathrm{g}$ of chromatin was incubated with antibodies directed against H3K4me3 (2 $\mu \mathrm{g}$, pAb-003-050, Diagenode) and H3K27ac ( $2 \mu \mathrm{g}$, ab4729, Abcam, Cambridge, UK) overnight at $4{ }^{\circ} \mathrm{C}$ or $2.5 \mu \mathrm{g}$ of chromatin was incubated with an anti-H3K4mel antibody (1 $\mu \mathrm{g}$, ab8895, Abcam) or an anti-H3K27me3 antibody (1 $\mu \mathrm{g}$, pAb-069050, Diagenode). Immunocomplexes were washed for $10 \mathrm{~min}$ each with buffer 1 (20 mM Tris-HCl, pH 8.0, $150 \mathrm{mM} \mathrm{NaCl}, 2 \mathrm{mM}$ EDTA, $1 \%$ Triton X-100, $0.1 \%$ SDS), buffer 2 (20 mM Tris- $\mathrm{HCl}, \mathrm{pH}$ 8.0, $500 \mathrm{mM} \mathrm{NaCl}, 2 \mathrm{mM}$ EDTA, $1 \%$ Triton $\mathrm{X}-100,0.1 \%$ SDS), buffer 3 (20 mM Tris-HCl, pH 8.0, $250 \mathrm{mM} \mathrm{LiCl,} 2 \mathrm{mM}$ EDTA $1 \%$ IGEPAL CA-630, $1 \%$ sodium deoxycholate) and three times with TE buffer (20 mM Tris-HCl, $\mathrm{pH} 8.0,2 \mathrm{mM}$ EDTA) at $4{ }^{\circ} \mathrm{C}$. Elution from beads was carried out by shaking in elution buffer $(100 \mathrm{mM} \mathrm{NaHCO} 3,1 \% \mathrm{SDS})$ at 1,400 r.p.m. at room temperature for $1 \mathrm{~h}$. DNA was treated with $50 \mathrm{ng} \mu \mathrm{l}^{-1}$ RNAse A (Sigma) for $30 \mathrm{~min}$ at $37^{\circ} \mathrm{C}$ and crosslinks were reversed by adding $0.5 \mu \mathrm{g} \mu \mathrm{l}^{-1}$ proteinase $\mathrm{K}$ (AppliChem, Darmstadt, Germany) and incubation at $65^{\circ} \mathrm{C}$ for $5 \mathrm{~h}$. DNA was purified using phenol/chloroform followed by ethanol precipitation at $-20^{\circ} \mathrm{C}$ overnight. DNA was quantified using Quant-iT PicoGreen dsDNA Reagent (Invitrogen). Sequencing libraries were prepared from $5 \mathrm{ng}$ DNA with the NEBNext ChIP-seq Library Prep Master Mix Set for Illumina (NEB) with 15 PCR cycles and sequenced on a HiSeq 2500 (50 bp, Illumina, San Diego, USA).

ChIP-seq data analysis. ChIP-seq reads were mapped to the $\mathrm{mm} 9$ genome assembly by Bowtie2 (ref. 68). For read coverage of input and histone modifications, the genome was binned. The number of reads per bin was counted and normalized to the total number of mappable reads (reads per kilobase per million mapped reads, RPKM). The enrichment of histone modifications in a given genomic region was calculated as the $\log 2$ ratio of ChIP RPKM and input RPKM. Peak calling was performed using MACS2 (ref. 69). 


\section{References}

1. Bergmann, O. et al. Evidence for cardiomyocyte renewal in humans. Science 324, 98-102 (2009).

2. Senyo, S. E. et al. Mammalian heart renewal by pre-existing cardiomyocytes. Nature 493, 433-436 (2013).

3. Taegtmeyer, H., Sen, S. \& Vela, D. Return to the fetal gene program: a suggested metabolic link to gene expression in the heart. Ann. NY Acad. Sci. 1188, 191-198 (2010).

4. Siedner, S. et al. Developmental changes in contractility and sarcomeric proteins from the early embryonic to the adult stage in the mouse heart. J. Physiol. 548, 493-505 (2003).

5. Krüger, M., Kohl, T. \& Linke, W. A. Developmental changes in passive stiffness and myofilament $\mathrm{ca} 2{ }^{+}$sensitivity due to titin and troponin-i isoform switching are not critically triggered by birth. Am. J. Physiol. Heart Circ. Physiol. 291, H496-H506 (2006).

6. Grueter, C. E. et al. A cardiac microrna governs systemic energy homeostasis by regulation of med13. Cell 149, 671-683 (2012).

7. Anand, P. et al. Bet bromodomains mediate transcriptional pause release in heart failure. Cell 154, 569-582 (2013)

8. Wamstad, J. A. et al. Dynamic and coordinated epigenetic regulation of developmental transitions in the cardiac lineage. Cell 151, 206-220 (2012).

9. Zhang, C. L. et al. Class II histone deacetylases act as signal-responsive repressors of cardiac hypertrophy. Cell 110, 479-488 (2002).

10. Hon, G. C. et al. Epigenetic memory at embryonic enhancers identified in DNA methylation maps from adult mouse tissues. Nat. Genet. 45, 1198-1206 (2013).

11. Bird, A. DNA methylation patterns and epigenetic memory. Genes Dev. 16, 6-21 (2002).

12. Jones, P. A. Functions of DNA methylation: islands, start sites, gene bodies and beyond. Nat. Rev. Genet. 13, 484-492 (2012).

13. Deaton, A. M. \& Bird, A. Cpg islands and the regulation of transcription. Genes Dev. 25, 1010-1022 (2011).

14. Smith, Z. D. \& Meissner, A. DNA methylation: Roles in mammalian development. Nat. Rev. Genet. 14, 204-220 (2013).

15. Reik, W., Dean, W. \& Walter, J. Epigenetic reprogramming in mammalian development. Science 293, 1089-1093 (2001).

16. Branco, M. R., Ficz, G. \& Reik, W. Uncovering the role of 5 hydroxymethylcytosine in the epigenome. Nat. Rev. Genet. 13, 7-13 (2012).

17. Haas, J. et al. Alterations in cardiac DNA methylation in human dilated cardiomyopathy. EMBO Mol. Med. 5, 413-429 (2013).

18. Movassagh, M. et al. Distinct epigenomic features in end-stage failing human hearts. Circulation 124, 2411-2422 (2011).

19. Rubart, M. \& Field, L. J. Cardiac regeneration: repopulating the heart. Annu. Rev. Physiol. 68, 29-49 (2006).

20. Bergmann, O. et al. Identification of cardiomyocyte nuclei and assessment of ploidy for the analysis of cell turnover. Exp. Cell Res. 317, 188-194 (2011).

21. Stadler, M. B. et al. DNA-binding factors shape the mouse methylome at distal regulatory regions. Nature 480, 490-495 (2011).

22. Ziller, M. J. et al. Charting a dynamic DNA methylation landscape of the human genome. Nature 500, 477-481 (2013).

23. Hnisz, D. et al. Super-enhancers in the control of cell identity and disease. Cell 155, 934-947 (2013).

24. Creyghton, M. P. et al. Histone h3k27ac separates active from poised enhancers and predicts developmental state. Proc. Natl Acad. Sci. USA 107, 21931-21936 (2010).

25. Zhuang, S. et al. Expression of isl1 during mouse development. Gene Expr. Patterns 13, 407-412 (2013).

26. Jiang, L. et al. Sperm, but not oocyte, DNA methylome is inherited by zebrafish early embryos. Cell 153, 773-784 (2013).

27. Srivastava, D. Making or breaking the heart: from lineage determination to morphogenesis. Cell 126, 1037-1048 (2006).

28. He, A. et al. Polycomb repressive complex 2 regulates normal development of the mouse heart. Circ. Res. 110, 406-415 (2012).

29. Delgado-Olguin, P. et al. Epigenetic repression of cardiac progenitor gene expression by ezh2 is required for postnatal cardiac homeostasis. Nat. Genet. 44, 343-347 (2012).

30. Walsh, S., Ponten, A., Fleischmann, B. K. \& Jovinge, S. Cardiomyocyte cell cycle control and growth estimation in vivo--an analysis based on cardiomyocyte nuclei. Cardiovasc. Res. 86, 365-373 (2010).

31. Hesse, M. et al. Direct visualization of cell division using high-resolution imaging of m-phase of the cell cycle. Nat. Commun. 3, 1076 (2012)

32. Feldmann, A. et al. Transcription factor occupancy can mediate active turnover of DNA methylation at regulatory regions. PLoS Genet. 9, e1003994 (2013).

33. Lister, R. et al. Global epigenomic reconfiguration during mammalian brain development. Science 341, 1237905 (2013).

34. Jeong, M. et al. Large conserved domains of low DNA methylation maintained by dnmt3a. Nat. Genet. 46, 17-23 (2013).

35. Xie, W. et al. Epigenomic analysis of multilineage differentiation of human embryonic stem cells. Cell 153, 1134-1148 (2013).
36. Lister, R. et al. Human DNA methylomes at base resolution show widespread epigenomic differences. Nature 462, 315-322 (2009).

37. Kobayashi, H. et al. Contribution of intragenic DNA methylation in mouse gametic DNA methylomes to establish oocyte-specific heritable marks. PLoS Genet. 8, e1002440 (2012).

38. Ball, M. P. et al. Targeted and genome-scale strategies reveal gene-body methylation signatures in human cells. Nat. Biotechnol. 27, 361-368 (2009).

39. Jermann, P., Hoerner, L., Burger, L. \& Schübeler, D. Short sequences can efficiently recruit histone h3 lysine 27 trimethylation in the absence of enhancer activity and DNA methylation. Proc. Natl Acad. Sci. USA 111, E3415-E3421 (2014).

40. Okano, M., Bell, D. W., Haber, D. A. \& Li, E. DNA methyltransferases dnmt3a and dnmt3b are essential for de novo methylation and mammalian development. Cell 99, 247-257 (1999).

41. Wettschureck, N. et al. Absence of pressure overload induced myocardial hypertrophy after conditional inactivation of galphaq/galpha11 in cardiomyocytes. Nat. Med. 7, 1236-1240 (2001).

42. Lother, A. et al. Ablation of mineralocorticoid receptors in myocytes but not in fibroblasts preserves cardiac function. Hypertension 57, 746-754 (2011).

43. Bratu, D. P., Cha, B. J., Mhlanga, M. M., Kramer, F. R. \& Tyagi, S. Visualizing the distribution and transport of mrnas in living cells. Proc. Natl Acad. Sci. USA 100, 13308-13313 (2003).

44. Ehler, E., Moore-Morris, T. \& Lange, S. Isolation and culture of neonatal mouse cardiomyocytes. J. Vis. Exp. doi: 10.3791/50134 (2013).

45. O’Connell, T. D., Rodrigo, M. C. \& Simpson, P. C. Isolation and culture of adult mouse cardiac myocytes. Methods Mol. Biol. 357, 271-296 (2007).

46. Shen, Y. et al. A map of the cis-regulatory sequences in the mouse genome. Nature 488, 116-120 (2012).

47. Blankenberg, D. et al. Galaxy: a web-based genome analysis tool for experimentalists. Curr. Protoc. Mol. Biol. Chapter 19: Unit 1910 11-Unit 1910 21 (2010).

48. Giardine, B. et al. Galaxy: a platform for interactive large-scale genome analysis Genome Res. 15, 1451-1455 (2005).

49. Goecks, J., Nekrutenko, A., Taylor, J. \& Galaxy, T. Galaxy: a comprehensive approach for supporting accessible, reproducible, and transparent computational research in the life sciences. Genome Biol. 11, R86 (2010).

50. Krueger, F. \& Andrews, S. R. Bismark: a flexible aligner and methylation caller for bisulfite-seq applications. Bioinformatics 27, 1571-1572 (2011).

51. Langmead, B., Trapnell, C., Pop, M. \& Salzberg, S. L. Ultrafast and memoryefficient alignment of short DNA sequences to the human genome. Genome Biol. 10, R25 (2009).

52. Li, H. et al. The sequence alignment/map format and samtools. Bioinformatics 25, 2078-2079 (2009).

53. Quinlan, A. R. \& Hall, I. M. Bedtools: a flexible suite of utilities for comparing genomic features. Bioinformatics 26, 841-842 (2010).

54. Robinson, J. T. et al. Integrative genomics viewer. Nat. Biotechnol. 29, 24-26 (2011).

55. Gentleman, R. C. et al. Bioconductor: open software development for computational biology and bioinformatics. Genome Biol. 5, R80 (2004).

56. Ramirez, F., Dundar, F., Diehl, S., Gruning, B. A. \& Manke, T. Deeptools: a flexible platform for exploring deep-sequencing data. Nucleic Acids Res. 42, W187-W191 (2014)

57. Heinz, S. et al. Simple combinations of lineage-determining transcription factors prime cis-regulatory elements required for macrophage and $\mathrm{b}$ cell identities. Mol. Cell 38, 576-589 (2010).

58. Royo, J. L., Hidalgo, M. \& Ruiz, A. Pyrosequencing protocol using a universal biotinylated primer for mutation detection and snp genotyping. Nat. Protoc. 2, 1734-1739 (2007).

59. Tost, J. \& Gut, I. G. DNA methylation analysis by pyrosequencing. Nat. Protoc. 2, 2265-2275 (2007)

60. McLean, C. Y. et al. Great improves functional interpretation of cis-regulatory regions. Nat. Biotechnol. 28, 495-501 (2010).

61. Bindea, G. et al. Cluego: a cytoscape plug-in to decipher functionally grouped gene ontology and pathway annotation networks. Bioinformatics 25, 1091-1093 (2009).

62. Wu, H., Caffo, B., Jaffee, H. A., Irizarry, R. A. \& Feinberg, A. P. Redefining cpg islands using hidden markov models. Biostatistics 11, 499-514 (2010).

63. Karolchik, D. et al. The ucsc genome browser database: 2014 update. Nucleic Acids Res. 42, D764-D770 (2014).

64. Kim, D. et al. Tophat2: accurate alignment of transcriptomes in the presence of insertions, deletions and gene fusions. Genome Biol. 14, R36 (2013)

65. Trapnell, C. et al. Transcript assembly and quantification by rna-seq reveals unannotated transcripts and isoform switching during cell differentiation. Nat Biotechnol. 28, 511-515 (2010).

66. Gilsbach, R. et al. Sympathetic alpha(2)-adrenoceptors prevent cardiac hypertrophy and fibrosis in mice at baseline but not after chronic pressure overload. Cardiovasc. Res. 86, 432-442 (2010). 
67. Bonn, S. et al. Cell type-specific chromatin immunoprecipitation from multicellular complex samples using bits-chip. Nat. Protoc. 7, 978-994 (2012).

68. Langmead, B. \& Salzberg, S. L. Fast gapped-read alignment with bowtie 2. Nat. Methods 9, 357-359 (2012).

69. Zhang, Y. et al. Model-based analysis of chip-seq (macs). Genome Biol. 9, R137 (2008).

\section{Acknowledgements}

We thank Claudia Köbele (University of Freiburg, Germany) for technical assistance, the European Molecular Biology Laboratory GeneCore, Genomics Core Facility staff (Heidelberg, Germany) for performing MethylC-seq and Julia K. Ellis for manuscript editing. This study was supported by the Deutsche Forschungsgemeinschaft SFB 992 project B03 (L.H.) and the BIOSS Centre for Biological Signalling Studies (L.H.).

\section{Author contributions}

R.G. and S.P. designed and performed the experiments. T.S. performed surgery and physiology. V.B. and U.B. performed sequencing and A.W. performed FACS. B.A.G. developed bioinformatic tools and B.A.G., R.G. and S.P. performed computational analysis. L.B., S.G., R.B. and B.K.F. interpreted the data and edited the manuscript. L.H. conceived the project and L.H., D.S., R.G. and S.P. wrote the manuscript.

\section{Additional information}

Accession codes: DNA methylomes, ChIP-Seq and RNA-seq files have been submitted to the NCBI BioSample databases under accessions SRP033288 (MethylC-seq), SRP033385 (ChIP-seq) and SRP033386 (RNA-seq).

Supplementary Information accompanies this paper at http://www.nature.com/ naturecommunications

Competing financial interests: The authors declare no competing financial interests.

Reprints and permission information is available online at http://npg.nature.com/ reprintsandpermissions/

How to cite this article: Gilsbach, R. et al. Dynamic DNA methylation orchestrates cardiomyocyte development, maturation and disease. Nat. Commun. 5:5288 doi: 10.1038/ncomms6288 (2014).

\section{(c) (i)}

This work is licensed under a Creative Commons Attribution 4.0 International License. The images or other third party material in this article are included in the article's Creative Commons license, unless indicated otherwise in the credit line; if the material is not included under the Creative Commons license, users will need to obtain permission from the license holder to reproduce the material. To view a copy of this license, visit http://creativecommons.org/licenses/by/4.0/ 